\title{
Bifurcation analysis and phase diagram of a spin-string model with buckled states
}

\author{
M. Ruiz-Garcia,,${ }^{1,2, *}$ L. L. Bonilla, ${ }^{1}$ and A. Prados ${ }^{3}$ \\ ${ }^{1}$ Gregorio Millán Institute for Fluid Dynamics, Nanoscience, and Industrial Mathematics, and Department of Materials Science and \\ Engineering and Chemical Engineering, Universidad Carlos III de Madrid, Avenida de la Universidad 30, 28911 Leganés, Spain \\ ${ }^{2}$ Department of Physics and Astronomy, University of Pennsylvania, Philadelphia, Pennsylvania 19104, USA \\ ${ }^{3}$ Física Teórica, Universidad de Sevilla, Apartado de Correos 1065, E-41080, Sevilla, Spain
}

(Received 11 August 2017; published 28 December 2017)

\begin{abstract}
We analyze a one-dimensional spin-string model, in which string oscillators are linearly coupled to their two nearest neighbors and to Ising spins representing internal degrees of freedom. String-spin coupling induces a long-range ferromagnetic interaction among spins that competes with a spin-spin antiferromagnetic coupling. As a consequence, the complex phase diagram of the system exhibits different flat rippled and buckled states, with first or second order transition lines between states. This complexity translates to the two-dimensional version of the model, whose numerical solution has been recently used to explain qualitatively the rippled to buckled transition observed in scanning tunneling microscopy experiments with suspended graphene sheets. Here we describe in detail the phase diagram of the simpler one-dimensional model and phase stability using bifurcation theory. This gives additional insight into the physical mechanisms underlying the different phases and the behavior observed in experiments.
\end{abstract}

DOI: 10.1103/PhysRevE.96.062147

\section{INTRODUCTION}

Rippling and buckling of suspended graphene sheets is an active research topic [1-9]. Recent scanning tunneling microscopy (STM) experiments show that local heating induces a transition from a soft rippled sheet to a hard buckled graphene membrane [9]. While heating certainly increases thermal fluctuations, in this case and quite counterintuitively, it produces a more ordered phase. We have interpreted the STM experiments as the result of driving the system through a first-order phase transition between flat and buckled membrane states [10]. We have used a phenomenological spin-membrane model of the graphene sheet that exhibits such a first-order phase transition [10]. In this model, the membrane is a system of mass points on a lattice that can move vertically and are interconnected by linear springs. At each lattice node, there is a pseudospin that represents in a simple way some internal degrees of freedom. The force due to the pseudospin pushes the point mass located at the same lattice site either upwards or downwards. In addition, nearest-neighbor pseudospins interact antiferromagnetically.

Despite the qualitative agreement between experiments and numerical simulations the main features of the phase diagram remained unclear. This work describes in detail the phase diagram of the 1D model, which presents the same features numerically observed in the 2D model. Bifurcation theory techniques allow us to give a clear explanation of every element in the phase diagram, including the already mentioned counterintuitive disorder to order rearrangement when temperature is increased.

Spin-string [11] and spin-membrane models $[6,10,12]$ simulate an elastic membrane or string coupled to internal degrees of freedom (the pseudospins). In the case of graphene, these internal variables may model the influence of charge density fluctuations that are coupled to the vertical deformations of

\footnotetext{
*miguelrg@sas.upenn.edu
}

the membrane $[8,13]$, and of atomic orbital hybridizations $\left(s p^{2}\right.$ to $\left.s p^{3}\right)[14,15]$. Our spin-membrane system mimics in a very simple way the effective interactions that appear after electrons and in-plane phonons are integrated out in electron-phonon models $[4,13]$. For instance, the antiferromagnetic interaction between pseudospins and the linear coupling between vertical displacements and pseudospins are basically the simplest possibilities for modeling the Coulomb interaction and the coupling between internal and elastic variables, respectively. The local pseudospin leads to the emergence of rippled and buckled phases, which is difficult to show using more microscopic approaches $[4,13]$. Thus spin-string and spin-membrane models should be considered useful toy models for graphene and other physical systems.

We expect these simplified models allow carrying out controlled analytical calculations and yet retain the main ingredients that make it possible to understand physically the observed behavior. We look for qualitative agreement with experiments, as a direct relation between the parameters of the model and physical constants of graphene has not been established. Although motivated by the non trivial mechanical properties of graphene, our results can be applied to any other system that may be modeled in a similar way. In other two-dimensional (2D) materials, polymer chains or biofilms, deformations are coupled to internal degrees of freedom and we could investigate their complex behavior along the lines presented in this work. We recall that related mechanical systems coupled to spins have been already used to describe structural phase transitions in other contexts [16-27].

Previous work has shown that pseudospins coupled only to mass points but not among themselves exhibit a second order buckling transition below a critical temperature [11]. A membrane described by Föppl-von Kármán equations on a hexagonal lattice with vertical displacements coupled to the local spin on the same lattice node displays the same behavior [6]. The second-order transition arises because the spin-membrane coupling produces a long range ferromagnetic 
interaction among pseudospins. This strengthens the physical relevance of simplifying the elastic part of the Hamiltonian.

Additional short-range antiferromagnetic couplings among the pseudospins produce different phases and first- or secondorder transitions among them [10,12]. One drawback of the 2D spin-membrane models with antiferromagnetic coupling is that most results are obtained from numerical simulations. In this work, we study analytically the corresponding onedimensional (1D) spin-string model, in terms of the dimensionless temperature $\theta$ and the spin-spin antiferromagnetic coupling $\kappa$. As already said above, for $\kappa=0$ there is a second-order phase transition at $\theta=1$ from a flat string configuration (stable for $\theta>1$ ) to stable buckled string states that exist for $\theta<1$. This second-order phase transition is a supercritical pitchfork bifurcation [28]. For $\kappa \neq 0$, we find and analyze subcritical pitchfork bifurcations corresponding to first-order phase transitions between flat and buckled phases. This situation is similar to the 2D case but, in $1 \mathrm{D}$, we are able to obtain bifurcation lines, bifurcation diagrams and the different phases by analytical methods. The order parameter spin magnetization acts as the norm of the solution in bifurcation diagrams [28].

The first-order phase transition $[29,30]$ occurring in this 1D spin-string model is akin to that found numerically in the 2D spin-membrane model. Our explanation of Schoelz et al.'s experiments [9] is that the STM drives the system dynamically across the first-order phase transition appearing in a certain range of antiferromagnetic coupling [10]. The same situation occurs in the 1D spin-string model but our analytical approach allows us to identify more clearly the physical mechanisms behind this behavior.

The paper is organized as follows. In Sec. II, we define the model and introduce the free energy density controlling its equilibrium behavior, together with the corresponding Euler-Lagrange equation governing the equilibrium profiles. Also, we briefly discuss the flat solution and its stability. Section III puts forward the main results of our study, including a discussion of main elements of the phase diagram of the system, leaving the derivations for the later sections. We analyze in detail the bifurcation from the flat solution in Sec. IV and the emergence of a (tri)critical point, at which the transition changes from second order to first order. In Sec. V, we study the low temperature limit of our system, focusing on the spin configurations underlying the parabolic profiles of the string. We present the main conclusions of our work in Sec. VI. The appendices deal with some technical details and calculations that are omitted in the main text.

\section{CONTINUUM LIMIT OF THE SPIN-STRING MODEL}

We consider a spin-string system with Hamiltonian

$$
\begin{aligned}
\mathcal{H}(\boldsymbol{u}, \boldsymbol{p}, \boldsymbol{\sigma})= & \sum_{j=0}^{N}\left[\frac{p_{j}^{2}}{2 m}+\frac{k}{2}\left(u_{j+1}-u_{j}\right)^{2}\right. \\
& \left.-f u_{j} \sigma_{j}+J \sigma_{j+1} \sigma_{j}\right] .
\end{aligned}
$$

Here, $u_{j}$ and $p_{j}, j=1, \ldots, N$ are the string vertical displacements and their conjugate momenta, respectively, and $\sigma_{j}= \pm 1$ are pseudospin variables $[11,12]$. The latter represent internal degrees of freedom arising from internal forces that push the atoms along the vertical direction. Therefore, we have: (i) a nearest-neighbor harmonic interaction between the elastic variables, $k\left(u_{j+1}-u_{j}\right)^{2}$, (ii) an on-site interaction between the elastic and the internal variables, $-f u_{j} \sigma_{j}$, and (iii) a nearest-neighbor spin-spin interaction, $J \sigma_{j+1} \sigma_{j}$. We have clamped boundary conditions at the string ends, $u_{0}=$ $p_{0}=\sigma_{0}=u_{N+1}=p_{N+1}=\sigma_{N+1}=0$.

This work focuses on equilibrium results, for nonequilibrium dynamics a natural option is that string variables $u_{j}$ and $p_{j}$ satisfy Hamilton's equations of motion, whereas the pseudospins $\sigma_{j}$ evolve following Glauber dynamics [31] at the thermal bath temperature $T$ [12]. Then the system reaches equilibrium in the long time limit. The probability density of finding the system in a certain configuration $(\boldsymbol{u}, \boldsymbol{p}, \boldsymbol{\sigma})$ is given by $e^{-\mathcal{H} / T} / Z$, where $Z$ is the partition function and we have set $k_{B}=1$. For $J=0$ and temperature below $T_{0}=\frac{f^{2} N^{2}}{k \pi^{2}}$, the system exhibits stable ripples [11]. We now make energy variables dimensionless by measuring them in units of $T_{0}$. The dimensionless coupling constant $\kappa$, temperature $\theta$, and displacement $u_{j}^{*}$ are

$$
\kappa=\frac{J}{T_{0}}, \quad \theta=\frac{T}{T_{0}}, \quad u_{j}^{*}=\frac{f u_{j}}{T_{0}} .
$$

Suitable units are introduced for the remaining variables. Henceforth, we drop the asterisk in the dimensionless displacement so as not to clutter our formulas. Further details about the possible scaling of the different parameters can be found in Ref. [12]. In particular, different scalings for $f$ and $k$ are possible. Depending on them, $T_{0}$ may be bounded or increase with system size. More experiments with crystal membranes such as graphene are necessary, varying both temperature and size, to obtain the correct scaling of the model parameters.

In this paper, we investigate the equilibrium states and the different phases of the model in the limit as $N \gg 1$ with $x=$ $i / N \in[0,1][12]$. We integrate out the pseudospins and the canonical momenta. Then the resulting equilibrium probability density $\mathcal{P}[u]$ of finding the string with a certain profile $u(x)$ is $\mathcal{P}[u ; \theta, \kappa] \propto \exp (-F[u ; \theta, \kappa] / \theta)$, in which

$$
\begin{aligned}
F[u ; \theta, \kappa]= & N \int_{0}^{1} d x f\left(u, u^{\prime} ; \theta, \kappa\right) \\
f\left(u, u^{\prime} ; \theta, \kappa\right)= & \frac{\left(u^{\prime}\right)^{2}}{2 \pi^{2}}-\theta \ln \zeta\left(\frac{u}{\theta}, \frac{\kappa}{\theta}\right) \\
\zeta\left(\frac{u}{\theta}, \frac{\kappa}{\theta}\right)= & \exp \left(-\frac{\kappa}{\theta}\right) \cosh \left(\frac{u}{\theta}\right) \\
& +\exp \left(\frac{\kappa}{\theta}\right) \sqrt{1+\exp \left(-\frac{4 \kappa}{\theta}\right) \sinh ^{2}\left(\frac{u}{\theta}\right)}
\end{aligned}
$$

In the equations above, $F[u ; \theta, \kappa], f\left(u, u^{\prime} ; \theta, \kappa\right)$, and $\ln \zeta(u ; \theta, \kappa)$ are the total free energy, the (local) free-energy density per unit length, and the logarithm of the pseudospins partition function per site, respectively. Note that, when integrating the pseudospins, the elastic deformation $u$ plays the role of an external field. In the continuum limit we are using, 
$u_{i+1}-u_{i}=O\left(N^{-1}\right)$, and thus one can use the analytical result for the partition function of an Ising chain in a uniform external field, as explained in detail in Ref. [12].

\section{A. Euler-Lagrange equation for the equilibrium profiles}

The equilibrium profiles $u_{\mathrm{eq}}(x)$ solve the Euler-Lagrange equation,

$$
\frac{1}{\pi^{2}} u_{\mathrm{eq}}^{\prime \prime}=-\mu\left(u_{\mathrm{eq}} ; \theta, \kappa\right), \quad u_{\mathrm{eq}}(0)=u_{\mathrm{eq}}(1)=0,
$$

where

$$
\mu(u ; \theta ; \kappa) \equiv-\frac{\partial f\left(u, u^{\prime} ; \theta, \kappa\right)}{\partial u}=\frac{e^{-\frac{2 \kappa}{\theta}} \sinh \left(\frac{u}{\theta}\right)}{\sqrt{e^{-\frac{4 \kappa}{\theta}} \sinh ^{2}\left(\frac{u}{\theta}\right)+1}},
$$

is the local value of the magnetization, $\left\langle\sigma_{i}\right\rangle \rightarrow \mu$ in the continuum limit. Clearly, the magnetization sets the local value of the string curvature.

Let us consider only the first buckled mode that has no internal nodes. The absolute value of the total magnetization distinguishes between buckled and flat profiles and it is therefore an order parameter,

$$
M(\theta ; \kappa)=\left|\int_{0}^{1} d x \mu\left(u_{\mathrm{eq}} ; \theta, \kappa\right)\right|
$$

Further information is given by incorporating the correlation between nearest-neighbor pseudospins. In the continuum limit, $\left\langle\sigma_{i} \sigma_{i+1}\right\rangle \rightarrow C$, and

$$
C(u ; \kappa, \theta) \equiv \frac{\partial f}{\partial \kappa}=-\theta \frac{\partial \ln \zeta}{\partial \kappa} .
$$

Now, we can define a domain length parameter,

$$
\mathcal{D} \mathcal{L}(\theta ; \kappa)=\frac{1}{2} \int_{0}^{1} d x\left[1+C\left(u_{\mathrm{eq}} ; \theta, \kappa\right)\right] .
$$

This domain length parameter is zero for perfect antiferromagnetic order, $1 / 2$ for a random configuration of the pseudospins, and 1 for perfect ferromagnetic order [12].

The free energy functional $F[u]$ has a relative (or weak) minimum for the curve $u=u_{\mathrm{eq}}(x)$ provided the following two conditions are satisfied:

(1) the curve $u_{\text {eq }}(x)$ must satisfy the Euler-Lagrange Eq. (4).

(2) The linearized Euler-Lagrange equation about $u_{\text {eq }}(x)$,

$$
\delta u^{\prime \prime}=-\pi^{2}\left(\frac{\partial \mu}{\partial u}\right)_{u=u_{\mathrm{eq}}} \delta u, \delta u(0)=\delta u(a)=0,
$$

must have only the trivial solution $\delta u(x) \equiv 0, \forall x$, for any $a \leqslant 1$.

Considered separately, each condition is necessary for $F[u]$ to have a weak minimum (with the nuance $a<1$ instead of $a \leqslant 1$ in the second one) [32].

\section{B. Flat string profile and its stability}

The flat string profile $u_{\mathrm{L}}(x) \equiv 0, \forall x$, is always a solution of the Euler-Lagrange equation, which we call phase L [33]. It is (locally) stable if it corresponds to a minimum of the freeenergy functional. For phase $\mathrm{L}$ and any $a \leqslant 1$, the boundary

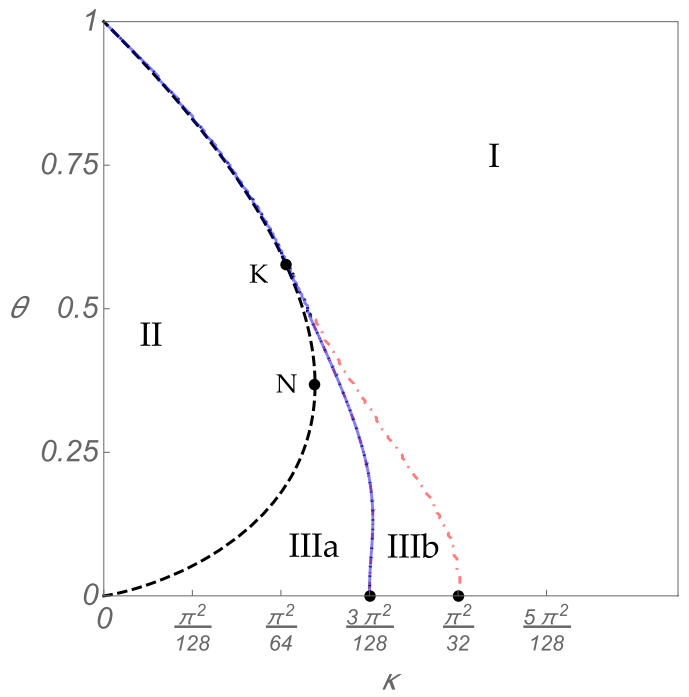

FIG. 1. Phase diagram in the $(\kappa, \theta)$ plane. We have marked the tricritical point $K$ and the turning point $N$ over the bifurcation curve $\kappa_{b}(\theta)$ (dashed line). Also plotted are the coexistence line $\kappa_{t}(\theta)$ (solid line) and the first-order line $\kappa_{M}(\theta)$ (dotted line). Note that $\kappa_{b}(\theta)<$ $\kappa_{t}(\theta)<\kappa_{M}(\theta)$. The definition of the different regions I, II, and III if the phase diagram, as well as the existing phases in each region and their stability is summarized in Table I. In addition, the values of $\kappa$ controlling the low-temperature behavior, $\kappa_{t}^{(0)}=3 \pi^{2} / 128$ and $\kappa_{M}^{(0)}=\pi^{2} / 32$, are shown with points.

value Eq. (9) is

$$
\delta u^{\prime \prime}=-\pi^{2} \theta^{-1} \exp (-2 \kappa / \theta) \delta u, u(0)=u(a)=0 .
$$

Aside from the trivial solution $\delta u(x) \equiv 0$, we may have solutions

$$
\delta u(x)=A \sin \left[\pi \theta^{-1 / 2} \exp (-\kappa / \theta) x\right],
$$

where $A$ is an arbitrary constant and $a$ is such that

$$
\theta^{-1 / 2} \exp (-\kappa / \theta) a=n, \quad n \in \mathbb{N}, a \leqslant 1 .
$$

Thus, first, the the flat solution produces a relative minimum of the free energy if $\theta^{-1 / 2} \exp (-\kappa / \theta)<1$. In this region of the $(\kappa, \theta)$ plane, the only solution of Eq. (10) is the trivial one. Second, if $\theta^{-1 / 2} \exp (-\kappa / \theta)>1$, there is at least one nontrivial solution of Eq. (10), provided we choose $a=\theta^{1 / 2} \exp (\kappa / \theta)<$ 1 and the flat profile is no longer stable.

Buckled equilibrium profiles may bifurcate at the curve $\theta^{1 / 2} \exp (\kappa / \theta)=1$, which is a bifurcation line in the $(\kappa, \theta)$ plane enclosing region II in Fig. 1. Points $\left(\kappa_{b}, \theta_{b}\right)$ on this line satisfy

$$
\theta_{b} \exp \left(\frac{2 \kappa_{b}}{\theta_{b}}\right)=1, \text { or } \kappa_{b}=-\frac{1}{2} \theta_{b} \ln \theta_{b} .
$$

The bifurcation line has two branches $\theta^{(2)}(\kappa)<\theta^{(1)}(\kappa)$ that coalesce at the turning point ("nose") $N \equiv\left(\kappa_{\mathrm{n}}=(2 e)^{-1}, \theta_{\mathrm{n}}=\right.$ $\left.e^{-1}\right), \theta_{\mathrm{n}}=2 \kappa_{\mathrm{n}}$. For $\kappa>\kappa_{\mathrm{n}}$, the free energy has a local minimum at the flat solution, regardless of the temperature. For $\kappa<\kappa_{\mathrm{n}}$ the flat solution is unstable if $\theta^{(2)}(\kappa)<\theta<\theta^{(1)}(\kappa)$, and locally stable otherwise; see Fig. 1 . Note that $\theta^{(2)}<2 \kappa<\theta^{(1)}$. The tangent to the bifurcation line at $\left(\kappa_{b}, \theta_{b}\right)$ verifies

$$
2 \delta \kappa_{b}+\left(1+\ln \theta_{b}\right) \delta \theta_{b}=0 .
$$


TABLE I. Summary of the different regions, phases (flat $L$, stable buckled $B+$ and unstable buckled $B-$ ), and their relative stability.

\begin{tabular}{lccccc}
\hline \hline \multirow{2}{*}{ Region } & Definition & Phases & \multicolumn{2}{c}{ Most } \\
stable & Unstable & Metastable \\
\hline I & $\frac{\kappa>\kappa_{b}(\theta) \theta>\theta_{c}}{\kappa>\kappa_{M}(\theta) \theta<\theta_{c}}$ & $\mathrm{~L}$ & $\mathrm{~L}$ & None & None \\
II & $\kappa<\kappa_{b}(\theta)$ & $\mathrm{B}+, \mathrm{L}$ & $\mathrm{B}+$ & $\mathrm{L}$ & None \\
IIIa & $\kappa_{b}(\theta)<\kappa<\kappa_{t}(\theta)$ & $\mathrm{B}+, \mathrm{B}-, \mathrm{L}$ & $\mathrm{B}+$ & $\mathrm{B}-$ & $\mathrm{L}$ \\
IIIb & $\kappa_{t}(\theta)<\kappa<\kappa_{M}(\theta)$ & $\mathrm{B}+, \mathrm{B}-, \mathrm{L}$ & $\mathrm{L}$ & $\mathrm{B}-$ & $\mathrm{B}+$ \\
\hline \hline
\end{tabular}

Here, $\delta \theta_{b}$ and $\delta \kappa_{b}$ are the (small) deviations from $\left(\kappa_{b}, \theta_{b}\right)$ over the tangent.

\section{RESULTS: PHASE DIAGRAM}

This section describes the main results of this paper, leaving derivations for later sections. There are three different phases in the system: the flat phase $L$ and two buckled phases, which we denote $B+$ and $B-$ (for low temperatures they are the string profiles shown in Fig. 5). The points and lines governing the existence and stability of the different phases are shown in Fig. 1. It shows the bifurcation line $\kappa_{b}(\theta)$ and the turning point $N$ that separates its two branches $\theta^{(1)}(\kappa) \geqslant \theta_{\mathrm{n}}$ and $\theta^{(2)}(\kappa) \leqslant \theta_{\mathrm{n}}$. The interior of the bifurcation curve is region II.

We shall now anticipate some results that will be discussed in depth in Sec. IV. A key element in the phase diagram is the existence of a tricritical point $K, K \equiv\left(\kappa_{c}=\sqrt{3} \ln 3 / 12, \theta_{c}=\right.$ $1 / \sqrt{3})$, at which the three phases $L, B+$ and $B-$ coalesce $[29,30]$. For $\theta>\theta_{c}$, the bifurcation at $\theta^{(1)}(\kappa)$ is supercritical, a stable buckled profile $B+$ stems continuously from the flat solution in region II. For $\theta<\theta_{c}$, the bifurcation becomes subcritical. Then an unstable buckled profile $B-$ issues from the flat solution at $\theta>\theta^{(1)}(\kappa)$ (upper bifurcation branch) and and at $\theta<\theta^{(2)}(\kappa)$ (lower branch). The stable buckled phase $B+$ does not disappear at $K$. Instead, $B+$ and the unstable state $B-$ coalesce at a temperature $\theta_{M}(\kappa)$ (dotted red line in Fig. 1) higher than $\theta^{(1)}(\kappa)$ for $\kappa>\kappa_{c}$. The transition at $K$ changes to first order. The phase $B+$ exists inside the bifurcation curve (region II) and also outside it (region III). For $\kappa>\kappa_{M}(\theta)$, we have only the flat phase $L$. In region III, there are three phases: $B-$ is unstable, whereas phases $L$ and $B+$ are both locally stable as they correspond to local minima of the free energy. Their relative stability depends on $\kappa$ : in fact, there appears a coexistence line $\kappa_{t}(\theta)$ (solid blue in Fig. 1) at which both phases are equiprobable. In region IIIa, $\kappa_{b}<\kappa<\kappa_{t}$, phase $B+$ provides the absolute minimum and phase $L$ is metastable, while in region IIIb, $\kappa_{t}<\kappa<\kappa_{M}$, the situation is reversed.

A summary of the above discussion is shown in Table I. Figures 2(a) and 2(b) show the bifurcation diagram of magnetization as a function of $\theta$ and $\kappa$. Figures 2(c)-2(f) depict the magnetization as a function of the temperature for several relevant values of $\kappa$. The bifurcation is always subcritical for the lower branch of the bifurcation curve. As $\kappa>0$ increases, the bifurcation at the upper branch changes from super to subcritical at the tricritical point $\kappa_{c} \simeq 0.159$. The two subcritical bifurcation points merge at the nose point $\kappa_{\mathrm{n}} \sim 0.184$. At higher $\kappa$, the buckled states give rise to an isola: these states become detached from the line $M=0$ and are thus isolated from the flat configuration. See two last panels in Fig. 2, corresponding to $\kappa>0.184$. The turning point $\theta_{M}(\kappa)$ at

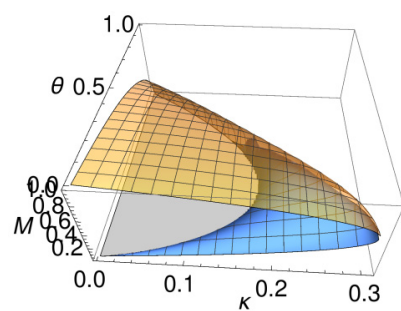

(a)

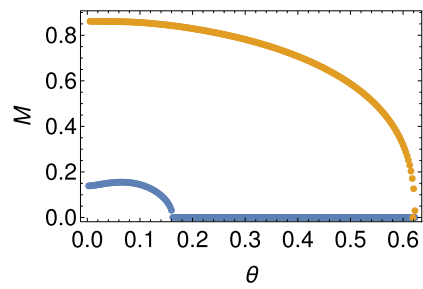

(c) $\kappa=0.15$

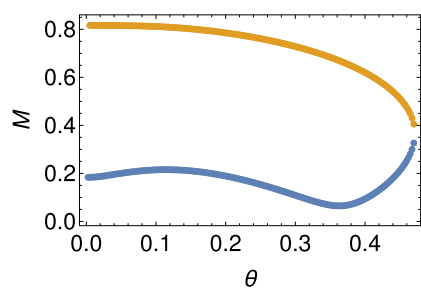

(e) $\kappa=0.185$

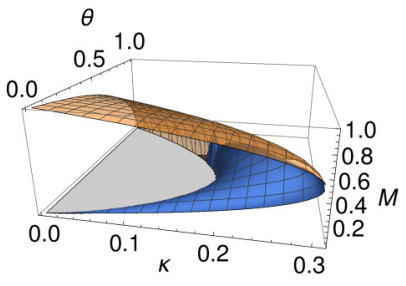

(b)

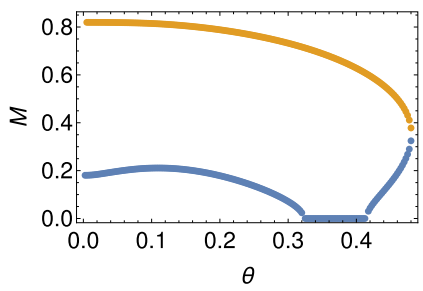

(d) $\kappa=0.18$

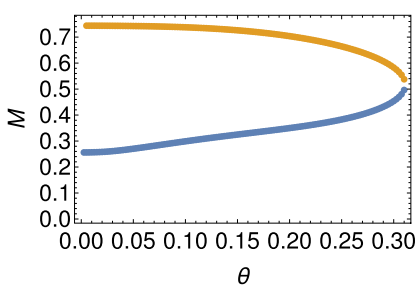

(f) $\kappa=0.24$
FIG. 2. Bifurcation diagrams numerically computed from Eq. (4). Panels (a) and (b) show the total magnetization of the buckled solutions versus $\kappa$ and $\theta$. The upper yellow (lower blue) surface stand for the stable (unstable) solution, two different profiles of such solutions at low temperatures can be found in Fig. 5. Panels (c)-(f) are bifurcation diagrams for increasing values of $\kappa$ depicting subcritical and supercritical bifurcations. For $\kappa>\kappa_{\mathrm{n}} \sim 0.184$, the subcritical bifurcations at the two branches of the bifurcation curve coalesce and an isola stems from the $M=0$ plane. Symmetric results with negative magnetization are omitted for clarity.

which buckled phases $B-$ and $B$ coalesce marks the boundary between regions IIIb and I. This is the first-order curve $\kappa_{M}(\theta)$.

A complementary description to bifurcation diagrams is given in Figs. 3 and 4. Figure 3 depicts the phase diagram of the model showed in Fig. 1 superimposed on the density plot of phase $B+$ magnetization (top panel) and free energy (bottom panel). In both panels, it is clearly observed the change of nature of the transition, from second to first order, at the tricritical point $K$. In the bottom panel, the change of relative stability between phases $B+$ and $L$ at the coexistence line $\kappa_{t}(\theta)$ is neatly seen, since $\Delta F$ vanishes. Figure 4 is completely analogous to Fig. 3 , but for the phase $B-$. Note that phase $B-$ only exists in region III and is always unstable, $\Delta F>0$ everywhere.

\section{BIFURCATIONS FROM THE FLAT STRING CONFIGURATION}

In this section, we calculate the buckled phases that issue from the flat string near the bifurcation line described in 

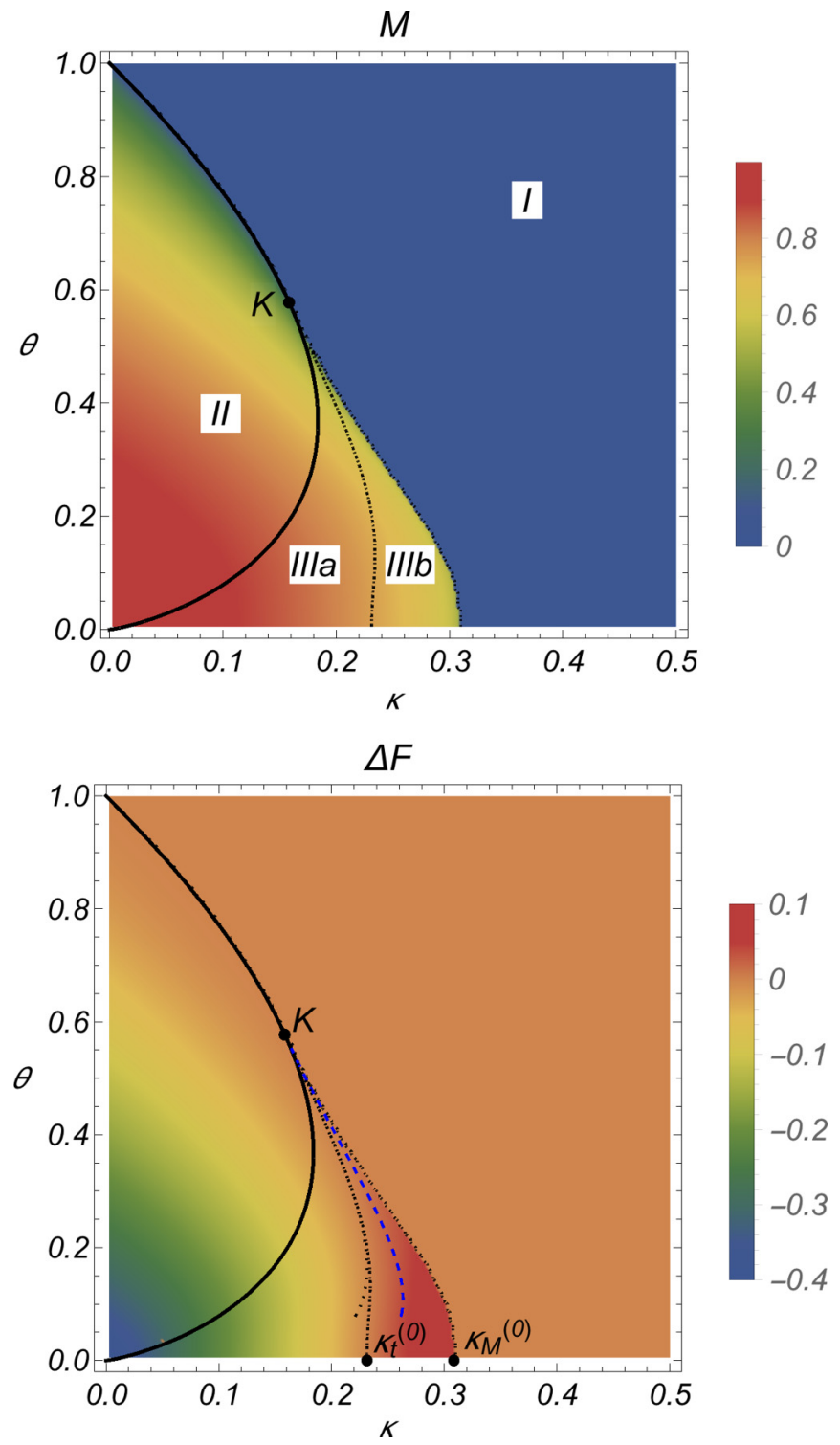

FIG. 3. Density plot of the magnetization $M$ (top panel) and the free energy difference $\Delta F$ (bottom panel) over the numerical solution of the Euler-Lagrange equation for the phase $\mathrm{B}+$. Also depicted are (i) the bifurcation line (solid) $\kappa_{b}(\theta)$, (ii) the coexistence line (dotted) $\kappa_{t}(\theta)$ that separates regions IIIa and IIIb, at which $\Delta F=0$, and (iii) the limit line $\kappa_{M}(\theta)$ (dotted). In the free-energy panel, we have also plotted the analytical expressions close to the critical point for the first-order transition lines $\kappa_{t}(\theta)$ (black dotted), Eq. (D12), and $\kappa_{M}(\theta)$ (dashed blue), Eq. (36). These theoretical lines are plotted up to $\theta=0.08$

Sec. III. Considerations on the stability of the phases are included in Appendix D.

\section{A. Pitchfork bifurcations from the flat string configuration}

First, we expand the free energy about the flat string configuration in powers of $u(x)=\epsilon U(x), \epsilon \ll 1$, and $U=$ $O(1)$, in which $\epsilon$ measures the amplitude of the string vertical displacement. We define the excess free energy density $\Delta f$ from the flat configuration (that has energy density $f_{L}$ )
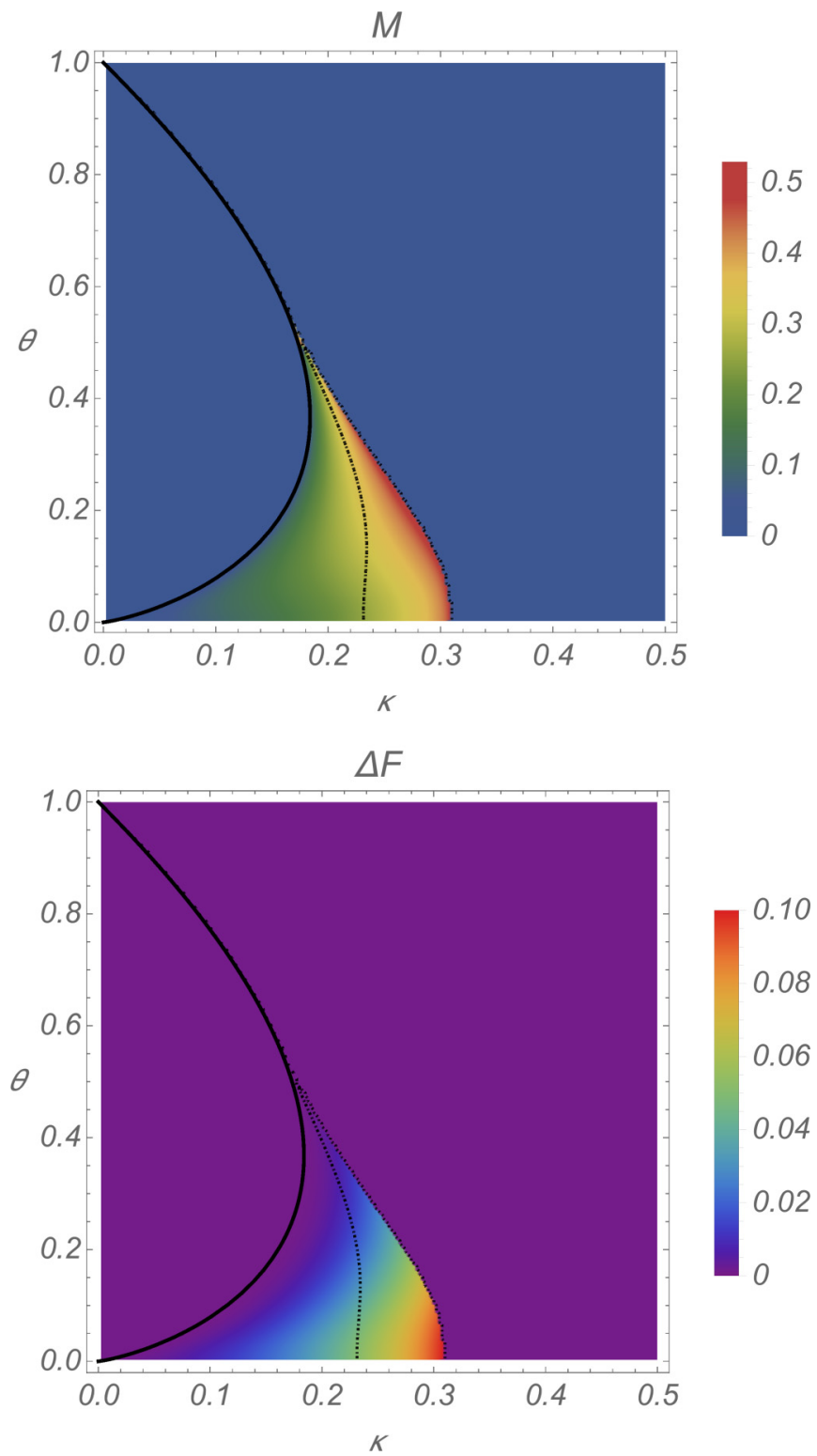

FIG. 4. Same as described in the caption of Fig. 3, but referred to unstable $B$ - buckled phase.

as

$$
\begin{gathered}
\Delta f\left(u, u^{\prime} ; \kappa, \theta\right) \equiv f\left(u, u^{\prime} ; \kappa, \theta\right)-f_{\mathrm{L}}(\kappa, \theta), \\
f_{\mathrm{L}}(\kappa, \theta) \equiv f(0,0 ; \kappa, \theta)=-\theta \ln \left(2 \cosh \frac{\kappa}{\theta}\right)
\end{gathered}
$$

This leads to

$$
\begin{aligned}
\Delta f\left(u, u^{\prime} ; \kappa, \theta\right)= & \frac{\epsilon^{2}}{2 \pi^{2}}\left(U^{\prime}\right)^{2}+\frac{\epsilon^{2}}{2 !} f_{2}(\kappa, \theta) U^{2} \\
& +\frac{\epsilon^{4}}{4 !} f_{4}(\kappa, \theta) U^{4}+\frac{\epsilon^{6}}{6 !} f_{6}(\kappa, \theta) U^{6}+O\left(\epsilon^{8}\right),
\end{aligned}
$$

in which

$$
\left.f_{n}(\kappa, \theta) \equiv \frac{\partial^{n} f\left(u, u^{\prime} ; \kappa, \theta\right)}{\partial u^{n}}\right|_{u=0}=-\frac{\partial^{n-1} \mu}{\partial u^{n-1}}(0 ; \kappa, \theta) .
$$


Here $\mu(u ; \kappa, \theta)=-\partial f / \partial u$ is the local magnetization. Using Eq. (5), we obtain

$$
\begin{aligned}
& f_{2}(\kappa, \theta)=-\frac{e^{-2 \kappa / \theta}}{\theta}, \\
& f_{4}(\kappa, \theta)=\frac{e^{-6 \kappa / \theta}}{\theta^{3}}\left(3-e^{4 \kappa / \theta}\right), \\
& f_{6}(\kappa, \theta)=-\frac{e^{-10 \kappa / \theta}}{\theta^{5}}\left(45-30 e^{4 \kappa / \theta}+e^{8 \kappa / \theta}\right) .
\end{aligned}
$$

The values of $f_{n}$ at the bifurcation line Eq. (13) are

$$
\begin{aligned}
f_{2, b} & =-1, \\
f_{4, b} & =\frac{3 \theta_{b}^{2}-1}{\theta_{b}^{2}}, \\
f_{6, b} & =\frac{-45 \theta_{b}^{4}+30 \theta_{b}^{2}-1}{\theta_{b}^{4}} .
\end{aligned}
$$

Second, we expand $\kappa$ and $\theta$ in powers of $\epsilon$ :

$$
\begin{aligned}
& \delta \kappa(\epsilon) \equiv \kappa(\epsilon)-\kappa_{b}=\epsilon^{2} \kappa_{2}+\epsilon^{4} \kappa_{4}+O\left(\epsilon^{6}\right), \\
& \delta \theta(\epsilon) \equiv \theta(\epsilon)-\theta_{b}=\epsilon^{2} \theta_{2}+\epsilon^{4} \theta_{4}+O\left(\epsilon^{6}\right) .
\end{aligned}
$$

The relation between $\delta \kappa$ and $\delta \theta$ fixes the direction in which we enter the different regions of the phase diagram. We anticipate that terms containing odd powers of $\epsilon$ vanish because $\Delta f$ is invariant under the transformation $U \rightarrow-U$.

We now expand $\Delta f$ up to $O\left(\epsilon^{4}\right)$ near the bifurcation line by inserting Eq. (21) into Eq. (19) and using Eq. (20a) with

$$
\delta f_{2, b} \equiv f_{2}(\kappa, \theta)-f_{2, b}=\frac{2}{\theta_{b}} \delta \kappa+\frac{1+\ln \theta_{b}}{\theta_{b}} \delta \theta .
$$

The result is

$$
\Delta f=\frac{\epsilon^{2}}{2}\left(\frac{U^{\prime 2}}{\pi^{2}}-U^{2}\right)+\epsilon^{4}\left(\frac{\varphi_{2}}{2} U^{2}+\frac{f_{4, b}}{24} U^{4}\right)+O\left(\epsilon^{6}\right),
$$

where

$$
\varphi_{j}=\frac{2 \kappa_{j}+\theta_{j}\left(1+\ln \theta_{b}\right)}{\theta_{b}} .
$$

The corresponding Euler-Lagrange equation, to be solved with clamped boundary conditions, is

$$
\frac{U^{\prime \prime}}{\pi^{2}}+U=\epsilon^{2}\left(\varphi_{2} U+\frac{f_{4, b}}{6} U^{3}\right)+O\left(\epsilon^{4}\right) .
$$

We now insert in this equation the ansatz

$$
U(x ; \epsilon)=U_{0}(x)+\epsilon^{2} U_{2}(x)+O\left(\epsilon^{4}\right) .
$$

All coefficients of powers of $\epsilon$ are zero separately, which supplies the hierarchy of equations

$$
\begin{aligned}
& \frac{U_{0}^{\prime \prime}}{\pi^{2}}+U_{0}=0, \\
& \frac{U_{2}^{\prime \prime}}{\pi^{2}}+U_{2}=\varphi_{2} U_{0}+\frac{f_{4, b}}{6} U_{0}^{3},
\end{aligned}
$$

and so on. The boundary conditions are $U_{j}(0)=U_{j}(1)=$ 0 . The solution of the first equation is $U_{0}(x)=A \sin \pi x$.
Equation (26b) has a solution with $U_{2}(0)=U_{2}(1)=0$ if its right-hand side (rhs) is orthogonal to $\sin \pi x$, that is,

$$
\int_{0}^{1} d x\left[\varphi_{2} U_{0}(x)+\frac{f_{4, b}}{6} U_{0}^{3}(x)\right] \sin \pi x=0 .
$$

This yields the bifurcation equation

$$
\varphi_{2} A+\frac{f_{4, b}}{8} A^{3}=0
$$

Its nonvanishing solutions obey

$$
0<A^{2}=-\frac{8 \varphi_{2}}{f_{4, b}}=-8 \theta_{b} \frac{2 \kappa_{2}+\theta_{2}\left(1+\ln \theta_{b}\right)}{3 \theta_{b}^{2}-1},
$$

provided $f_{4, b} \neq 0\left(\theta_{b} \neq \theta_{c}\right)$. In Eq. (29) we have substituted $\varphi_{2}$ and $f_{4, b}$ by their explicit expressions.

Let $\kappa$ be the bifurcation parameter, so that $\theta_{2}=0$. For $\theta_{b}>\theta_{c}$, Eq. (29) produces $\kappa_{2}<0$. Then $\kappa<\kappa_{b}$, and the buckled phases exist only inside region II of Fig. 1, where the flat string is unstable, i.e., the bifurcation is supercritical. For $\theta_{b}<\theta_{c}$ (which also occurs at the whole lower branch of the bifurcation line), we obtain $\kappa_{2}>0$, so that $\kappa>\kappa_{b}$. The buckled phase bifurcates outside region II where the flat string is stable, i.e., the bifurcation is subcritical. Clearly the bifurcating branches scale as $\left|\kappa-\kappa_{b}\right|^{1 / 2}$, the usual scaling for a pitchfork bifurcation.

\section{B. Bifurcation at the tricritical point}

At the tricritical point $K$, the coefficient of $A^{3}$ in the bifurcation Eq. (28) vanishes. We can unfold this bifurcation by expanding the free energy up to $O\left(\epsilon^{6}\right)$ terms [34,35] and rescaling the bifurcation parameters. If we set $\theta_{b}=\theta_{c}+\epsilon^{2} \chi$, with $\chi=O(1), f_{4, b}=O\left(\epsilon^{2}\right)$. Then the leading terms of the coefficients of $U^{4}$ and $U^{6}$ in $\Delta f$ are both $O\left(\epsilon^{6}\right)$. Assuming that $\delta \kappa$ and $\delta \theta$ are also $O\left(\epsilon^{4}\right)\left(\kappa_{2}=\theta_{2}=0\right), \epsilon^{2} \delta f_{2, b} U^{2}=O\left(\epsilon^{6}\right)$. Then,

$$
\begin{aligned}
& \theta=\theta_{b}+\epsilon^{4} \theta_{4}=\theta_{c}+\epsilon^{2} \chi+\epsilon^{4} \theta_{4}+O\left(\epsilon^{6}\right), \\
& \kappa=\kappa_{b}+\epsilon^{4} \kappa_{4}+O\left(\epsilon^{6}\right) .
\end{aligned}
$$

Keeping terms up to $O\left(\epsilon^{6}\right)$, we obtain

$$
\Delta f=\frac{\epsilon^{2}}{2}\left[\frac{\left(U^{\prime}\right)^{2}}{\pi^{2}}-U^{2}\right]+\epsilon^{6}\left[\frac{\varphi_{4, c}}{2} U^{2}+\frac{\sqrt{3} \chi}{4} U^{4}+\frac{U^{6}}{20}\right],
$$

where we have omitted $O\left(\epsilon^{8}\right)$ terms and introduced the notation

$$
\left.\varphi_{4, c} \equiv \varphi_{4}\right|_{\theta_{b}=\theta_{c}}=\frac{\sqrt{3}}{2}\left[4 \kappa_{4}+\theta_{4}(2-\ln 3)\right] .
$$

The corresponding Euler-Lagrange equation is

$$
\frac{U^{\prime \prime}}{\pi^{2}}+U=\epsilon^{4}\left[\varphi_{4, c} U+\sqrt{3} \chi U^{3}+\frac{3}{10} U^{5}\right]+O\left(\epsilon^{6}\right),
$$

to be solved with clamped boundary conditions. We now insert in this equation the ansatz

$$
U(x ; \epsilon)=U_{0}(x)+\epsilon^{4} U_{4}(x)+O\left(\epsilon^{6}\right),
$$


thereby obtaining a hierarchy of equations. The equation for $U_{0}$ is the same as before, whereas $U_{4}$ solves

$$
\frac{U_{4}^{\prime \prime}}{\pi^{2}}+U_{4}=\varphi_{4, c} U_{0}+\sqrt{3} \chi U_{0}^{3}+\frac{3}{10} U_{0}^{5} .
$$

The condition that the rhs of this equation be orthogonal to $\sin \pi x$ produces the equation for $A$. For $A \neq 0$, it is

$$
A^{4}+4 \sqrt{3} \chi A^{2}+\frac{8}{\sqrt{3}}\left[4 \kappa_{4}+\theta_{4}(2-\ln 3)\right]=0 .
$$

Here we have substituted the explicit expression for $\varphi_{4, c}$.

Let us analyze the solutions of Eq. (35) for $\theta_{4}=0$. Then $A$ is a function of $\chi$ and $\kappa_{4}$. In Fig. 1 , the system is just above (below) of the critical point for $\chi>0(\chi<0)$ and just outside (inside) the bifurcation curve for $\kappa_{4}>0\left(\kappa_{4}<\right.$ 0 ). For $\chi>0$, Eq. (35) has one positive solution $A^{2}>0$ if $\kappa_{4}<0\left(A^{2}=0\right.$ for $\left.\kappa_{4}=0\right)$. No real solutions exist if $\kappa_{4}>$ 0 . For $\chi<0$, Eq. (35) has one positive solution $A^{2}>0$ if $\kappa_{4}<0$ (corresponding to the stable phase $B+$ ). Depending on the sign of the discriminant of the biquadratic equation, Eq. (35) has two or zero positive solutions $A^{2}>0$ for $\kappa_{4}>0$ (corresponding to stable and unstable phases $B+$ and $B-$ ). The discriminant of Eq. (35) vanishes at the curve

$$
\kappa_{M}(\theta)=\kappa_{b}(\theta)+\frac{3 \sqrt{3}}{8}\left(\theta-\theta_{c}\right)^{2} .
$$

Specifically, there are two solutions for $\kappa<\kappa_{M}(\theta)$, denoted by $A_{ \pm}^{2}, A_{-}^{2}<A_{+}^{2}$, and no solutions for $\kappa>\kappa_{M}(\theta)$. For $\kappa<\kappa_{M}$, the solution $A_{-}$corresponds to phase $B-$ and it issues from the flat configuration as an unstable subcritical bifurcation at $\theta=\theta_{b}$. The solution $A_{+}$corresponds to phase $B+$, and it matches at $\theta_{c}$ the only unique phase existing for $\theta>\theta_{c}$. At the line $\kappa_{M}(\theta)$, phases $B-$ and $B+$ coalesce and disappear, which is consistent with the physical picture of a first-order phase transition. For more details, see Appendix D.

Note that Eq. (35) becomes

$$
A^{2} \sim-\frac{2}{3 \chi}\left[4 \kappa_{4}+\theta_{4}(2-\ln 3)\right],
$$

as $\chi \gg 1$. This relation follows from Eq. (29) if we substitute $\theta_{b}=\theta_{c}+\epsilon^{2} \chi, \theta_{2}=\epsilon^{2} \theta_{4}$ and $\kappa_{2}=\epsilon^{2} \kappa_{4}$ therein. Therefore, as expected, the bifurcating solution of Eq. (35) matches the solution of the bifurcation Eq. (29) as we move away from the tricritical point.

\section{Bifurcation at the turning point}

At the turning point $N$, the coefficient of $A$ in the bifurcation Eq. (28) becomes $2 e \kappa_{2}$, independent of $\theta_{2}$. We can unfold this bifurcation by rescaling the bifurcation parameter

$$
\kappa=\underbrace{\frac{1}{2 e}}_{\kappa_{\mathrm{n}}}+\epsilon^{4} \kappa_{4}+O\left(\epsilon^{6}\right)
$$

and expanding the coefficient of $U^{2}$ in the free energy up to $O\left(\epsilon^{4} \theta_{2}^{2}\right)$ terms. Inserting the result in Eq. (23), we obtain

$$
\begin{aligned}
\Delta f= & \frac{\epsilon^{2}}{2}\left[\frac{\left(U^{\prime}\right)^{2}}{\pi^{2}}-U^{2}\right] \\
& +\epsilon^{4}\left[\left(\kappa_{4}+\frac{e}{4} \theta_{2}^{2}\right) e U^{2}+\frac{3-e^{2}}{24} U^{4}\right]+O\left(\epsilon^{6}\right) .
\end{aligned}
$$

The corresponding Euler-Lagrange equations are

$$
\frac{U^{\prime \prime}}{\pi^{2}}+U=\epsilon^{2}\left[2 e\left(\kappa_{4}+\frac{e}{4} \theta_{2}^{2}\right) U-\frac{e^{2}-3}{6} U^{3}\right]+O\left(\epsilon^{4}\right),
$$

to be solved with clamped boundary conditions. Inserting Eq. (25) into this formula and equating like powers of $\epsilon$, we obtain a hierarchy of equations.

Again, the solution of the first equation of the hierarchy with clamped boundary conditions is $U_{0}(x)=A \sin \pi x$. The second equation is

$$
\frac{U_{2}^{\prime \prime}}{\pi^{2}}+U_{2}=2 e\left(\kappa_{4}+\frac{e}{4} \theta_{2}^{2}\right) U_{0}-\frac{e^{2}-3}{6} U_{0}^{3} .
$$

This equation has a solution that satisfies clamped boundary conditions provided its rhs is orthogonal to $\sin \pi x$, which yields

$$
2 e\left(\kappa_{4}+\frac{e}{4} \theta_{2}^{2}\right) A-\frac{e^{2}-3}{8} A^{3}=0 .
$$

The nontrivial solution of this equation satisfies

$$
0<A^{2}=\frac{4 e\left(4 \kappa_{4}+e \theta_{2}^{2}\right)}{e^{2}-3} .
$$

Note that $\kappa_{4}=-e \theta_{2}^{2} / 4$ is nothing but the lowest approximation to the bifurcation curve in the vicinity of the nose, written in the scaled variables.

Equation (40) implies that buckled solutions stem continuously from the parabola $\kappa_{4}=-e \theta_{2}^{2} / 4$ and exist outside it. These buckled states (corresponding to phase $B-$ ) bifurcate subcritically at $\theta_{2}^{(1,2)}= \pm 2 \sqrt{-\kappa_{4} / e}$. The corresponding temperatures are on the upper and lower branches of the bifurcation curve, respectively. At the turning point $\kappa_{4}=0$ and the two bifurcation points merge. For $\kappa_{4}>0\left(\kappa>\kappa_{\mathrm{n}}\right)$, there is a single unstable buckled state given by Eq. (40), for points close enough to the bifurcation curve.

Note that the stable phase $B+$ cannot be predicted by the bifurcation analysis near the nose, since the corresponding string profile is not close to the flat solution therein. We know that, for fixed $\kappa>\kappa_{\mathrm{n}}$, both buckled phases $B \pm$ coalesce at the boundary between regions IIIb and I in Fig. 3. These buckled string configurations persist as the temperature $\theta \rightarrow 0+$ for all spin-spin couplings $\kappa<\kappa_{M}^{(0)}=\pi^{2} / 32$, as indicated in Sec. V A.

\section{LOW-TEMPERATURE BEHAVIOR}

\section{A. Low-temperature profiles: Exact solution of the Euler-Lagrange equation}

At very low temperatures, such that $\exp (-2 \kappa / \theta) / \theta<1$ in Fig. 1, there are buckled solutions in addition to the stable flat profile. We calculate exactly their profiles below. In fact, for $\theta \ll|u|$, the local magnetization $\mu$ of Eq. (5) and the nearest-neighbor correlation $C$ of Eq. (7) reduce to

$$
\begin{aligned}
& \mu\left(u ; \kappa, \theta=0^{+}\right)=\operatorname{sgn}(u) \eta(|u|-2 \kappa), \\
& C\left(u ; \kappa, \theta=0^{+}\right)=\operatorname{sgn}(|u|-2 \kappa) .
\end{aligned}
$$

Here $\eta(x)$ is the Heaviside step function and $\operatorname{sgn}(x)=2 \eta(x)-$ 1 is the sign function. 


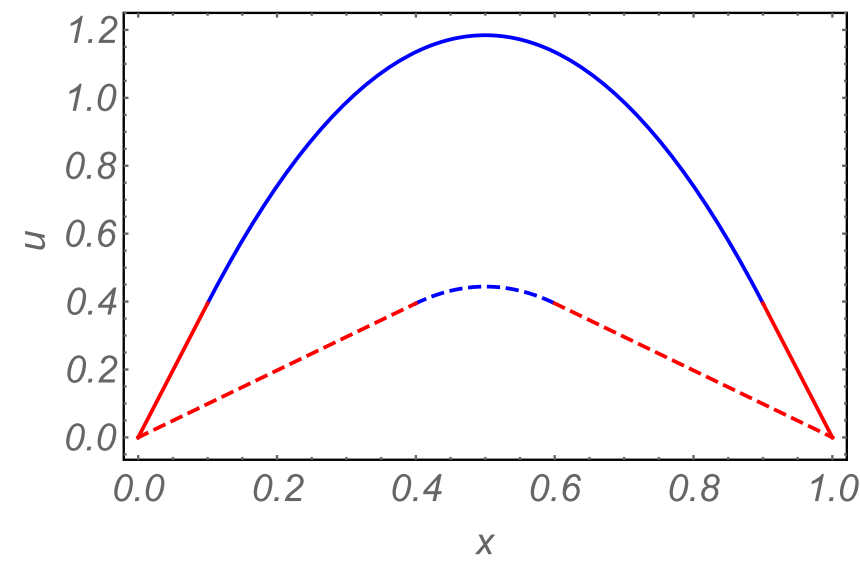

FIG. 5. Low-temperature nonflat string profiles. They comprise two linear zones of width $x_{0}$ near the endpoints of the chain (in red) and a parabolic zone in the middle of the system of width $1-2 x_{0}$ (in blue). Spins are ordered antiferromagnetically in the linear zones and ferromagnetically in the central parabolic zone; see Fig. 6. In this plot, $\kappa=\pi^{2} / 50<\kappa_{t}^{(0)}$, which gives two possible values of $x_{0}$ : $x_{0,1}=1 / 10$ (solid line) and $x_{0,2}=2 / 5$ (dashed line). The buckled profile corresponding to $x_{0,2}$ is always unstable. The string profile corresponding to $x_{0,1}<1 / 8$ gives the absolute minimum of the free energy, and the flat string is metastable.

Substitution of Eq. (41a) into Eq. (4) leads to $u^{\prime \prime}=0$ if $|u|<u_{0}=2 \kappa$ and $u^{\prime \prime}= \pm \pi^{2}$ if $|u|>2 \kappa$. Then $u(x)$ is a linear function if $|u|<2 \kappa$, and a parabola if $|u|>2 \kappa$. Due to the clamped boundary conditions, buckled solutions with a single extremum (no internal nodes) are linear close to the boundaries, $x \in\left(0, x_{0}\right)$ or $x \in\left(1-x_{0}, 1\right)$, and have a parabolic profile in the bulk $x \in\left(x_{0}, 1-x_{0}\right)$; see also Appendix A. Moreover, Eq. (41b) predicts that the nearest-neighbor correlation for the pseudospins is $C=-1$, close to the boundaries and $C=+1$ in the bulk. The condition $\left|u\left(x_{0}\right)\right|=2 \kappa$ produces the condition $\pi^{2} x_{0}\left(1-2 x_{0}\right)=4 \kappa$ whose solutions $x_{0,1}$ and $x_{0,2}$ are

$$
x_{0, j}=\frac{1}{4}\left(1+(-1)^{j} \sqrt{1-\frac{\kappa}{\kappa_{M}^{(0)}}}\right), \quad j=1,2,
$$

for $\kappa<\kappa_{M}^{(0)}=\pi^{2} / 32$. We have $x_{0,1}<1 / 4<x_{0,2}, x_{0,1}+$ $x_{0,2}=1 / 2$. If $\kappa>\kappa_{M}^{(0)}$, then these rippled low-temperature profiles are not possible and the only solution is $u=0$.

Figure 5 shows two of these profiles for an appropriate value of $\kappa$. The same functions multiplied by -1 are also stationary solutions. In these string profiles, the pseudospins exhibit antiferromagnetic order close to the boundaries and ferromagnetic order in the bulk, as predicted above and further discussed below in Sec. V B.

The profiles with $x_{0,1}<1 / 4$ produce a relative minimum of the free energy and are stable whereas those with $x_{0,2}>1 / 4$ are unstable [36], as proven in Appendix A. Thus, for $\kappa<$ $\kappa_{M}^{(0)}$, the buckled profiles with $x_{0,1}<1 / 4$ and the flat string are stable and the unstable profiles with $x_{0,2}>1 / 4$ separate them. The stable and unstable buckled profiles coalesce and disappear at $\kappa=\kappa_{M}^{(0)}\left(x_{0,1}=x_{0,2}=1 / 4\right)$. This allows us to identify the buckled profiles with $x_{0,1}$ and $x_{0,2}$ as the low temperature limits of phases $B+$ and $B-$, respectively.
By direct integration, we can show that the absolute minimum of the free energy corresponds to the buckled configurations with $x_{0,1}$ if $0<\kappa<\kappa_{t}^{(0)}=3 \pi^{2} / 128(0<$ $\left.x_{0,1}<x_{t}=1 / 8\right)$. For $\kappa_{t}^{(0)}<\kappa<\kappa_{M}^{(0)}\left(x_{t}<x_{0,1}<1 / 4\right)$, the free energy of the flat string is smaller than that of the buckled configurations with $x_{0}=x_{0,1}$. Thus, the flat string profile is metastable for $0<\kappa<\kappa_{t}^{(0)}$ and stable for $\kappa_{t}^{(0)}<\kappa<\kappa_{M}^{(0)}$. The situation is reversed for the buckled configurations with $x_{0}=x_{0,1}$. At $\kappa=\kappa_{t}^{(0)}$ there is a first-order phase transition, where the buckled phase with $x_{01}$ and the flat string coexist. Consistently, the first-order derivatives of the free energy change discontinuously at $\kappa=\kappa_{t}^{(0)}$. In fact, as $\kappa$ increases past $\kappa_{t}^{(0)}, M$ and $\mathcal{D} \mathcal{L}$ jump from $M=3 / 4$ and $\mathcal{D} \mathcal{L}=3 / 4$ (buckled phase with $x_{0,1}$ ) to $M=0$ and $\mathcal{D} \mathcal{L}=1 / 2$ (flat phase).

\section{B. Spin configurations of the low-temperature buckled string states}

What are the spin configurations at buckled string states? It turns out that the spins form antiferromagnetic domains near the boundaries and ferromagnetic domains in the central region of the string. To see this, we derive their marginal probability $\mathcal{P}(\sigma)$ by integrating the canonical distribution $\exp (-\mathcal{H} / T)$ over the string degrees of freedom. The result is

$$
\mathcal{P}(\boldsymbol{\sigma}) \propto e^{-\mathcal{H}_{\mathrm{eff}}(\boldsymbol{\sigma}) / \theta}, \quad \mathcal{H}_{\mathrm{eff}}(\boldsymbol{\sigma})=\kappa \boldsymbol{\sigma}^{\boldsymbol{T}} \boldsymbol{J} \boldsymbol{\sigma}-\frac{\pi^{2}}{2 N^{2}} \boldsymbol{\sigma}^{\boldsymbol{T}} \boldsymbol{\Lambda} \boldsymbol{\sigma} .
$$

Here, the effective spin Hamiltonian $\mathcal{H}_{\text {eff }}$ contains a nearest neighbor antiferromagnetic interaction given by

$$
\boldsymbol{J}_{i j}=\frac{1}{2}\left(\delta_{i, j+1}+\delta_{i, j-1}\right)
$$

and a long-ranged ferromagnetic interaction given by

$$
\boldsymbol{\Lambda}_{i j}=\frac{1}{N+1} j(N-i+1)>0, \quad \forall i \geqslant j, \quad \boldsymbol{\Lambda}_{i j}=\boldsymbol{\Lambda}_{j i},
$$

which is derived in Appendix B. Phase transitions in a one-dimensional model stem from this effective long range interaction, similarly to the situation found in other spinoscillator models $[11,16,17]$.

We focus on the low-temperature limit as $\theta \rightarrow 0^{+}$: therein, the equilibrium probability concentrates in the spin configuration that corresponds to the absolute minimum of $H_{\text {eff }}$. The long-range ferromagnetic interaction Eq. (45) is stronger for the pseudospins located near the center of the system than for those close to the boundaries. Therefore, as the intensity of the antiferromagnetic interaction $\kappa$ increases, the absolute minimum of $H_{\text {eff }}$ moves from the completely ferromagnetic configuration to one that is antiferromagnetic at the boundaries and ferromagnetic in the bulk. See Appendix C for details.

In light of the previous discussion, we restrict ourselves to states that are antiferromagnetic at the boundaries and ferromagnetic in the center. Note that this restriction includes completely antiferromagnetic and ferromagnetic states. We label the states by the number $n_{a}=1,3,5, \ldots, N / 2$ of spins at the antiferromagnetic boundary regions; see Fig. 6. Moreover, we denote by $\mathcal{H}_{\text {eff }}\left(n_{a}\right)$ the effective potential for such a 


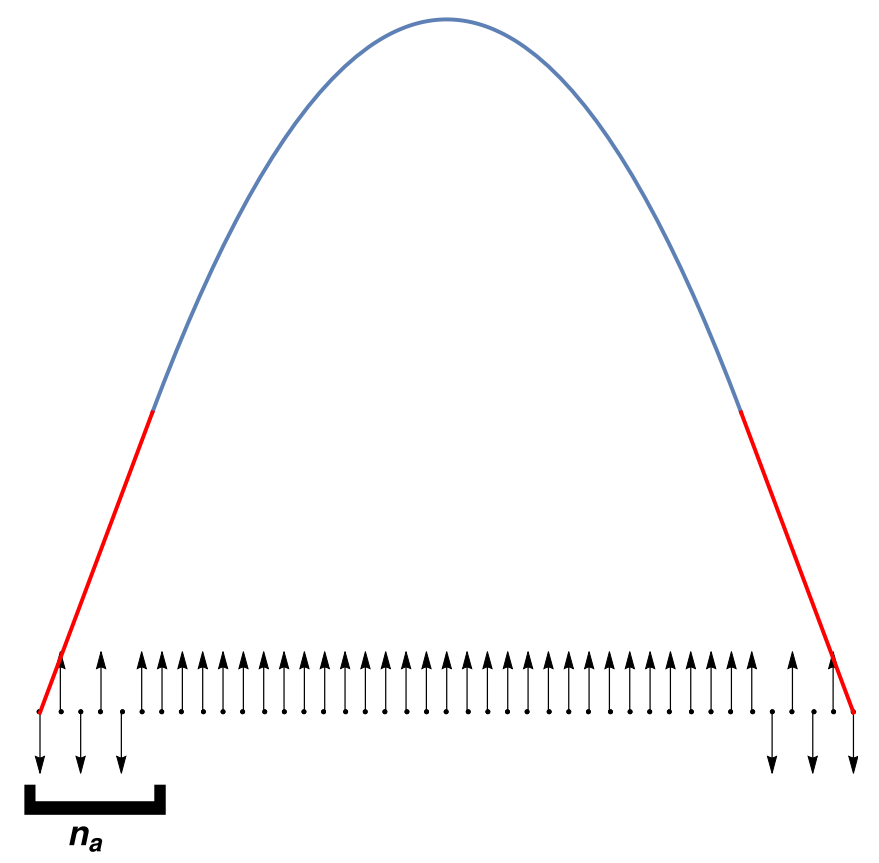

FIG. 6. Qualitative graph for the typical low-temperature configurations for the pseudospins and the string. In the antiferromagnetic regions close to the boundaries, there is no net magnetization and thus the string has a linear profile $\left(u^{\prime \prime}=0\right.$, red). In the ferromagnetic region in the bulk, the string takes a parabolic shape $\left(u^{\prime \prime}=-1\right.$, blue). We are plotting a system with $N=41$ pseudospins and $n_{a}=5$, which is the number of antiferromagnetic links at either boundary.

configuration. In Appendix C, we find

$$
\begin{aligned}
\mathcal{H}_{\mathrm{eff}}\left(n_{a}\right)= & \left(n_{a}-1\right)\left\{\frac { \pi ^ { 2 } } { 6 N ^ { 2 } } \left[N\left(3+n_{a}\right) 3-21-13 n_{a}\right.\right. \\
& \left.\left.-4 n_{a}^{2}\right]-4 \kappa\right\} .
\end{aligned}
$$

The origin of energy is such that $\mathcal{H}_{\mathrm{eff}}\left(n_{a}=1\right)=0$.

Depending on the value of $\kappa, \mathcal{H}_{\mathrm{eff}}\left(n_{a}\right)$ has one or two minima, as seen in Fig. 7. For $\kappa=0$, the completely ferromagnetic configuration gives the minimum of $H_{\text {eff }}$, as expected on physical grounds. On the other hand, as $\kappa$ increases, there appear several relevant values of $\kappa$, namely

$$
\begin{aligned}
\kappa_{0} & =\frac{\pi^{2}}{4} \frac{N-1}{N(N+1)}, \\
\kappa_{1} & =\frac{\pi^{2}}{384} \frac{9 N^{2}+6 N-47}{N^{2}}, \\
\kappa_{2} & =\frac{\pi^{2}}{96} \frac{3 N^{2}+6 N-5}{N^{2}},
\end{aligned}
$$

the physical meaning of which are discussed below. First, for $\kappa=\kappa_{0}$, the configurations with $n_{a}=1$ and $n_{a}=0$ share the same value of $\mathcal{H}_{\text {eff }}$. This marks the onset of the antiferromagnetic ordering at the boundaries, although for a large system this ordering is only relevant when $n_{a} / N$ becomes of the order of unity. In fact, for large $N, \kappa_{0}$ is proportional to $N^{-1}$, whereas both $\kappa_{1}$ and $\kappa_{2}$ become independent of $N$. Second, at $\kappa_{1}$, the relative minimum of $\mathcal{H}_{\text {eff }}$ has the

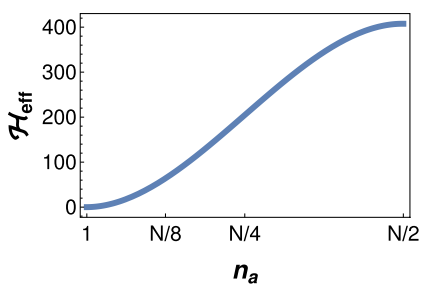

(a) $\kappa=\kappa_{0}$

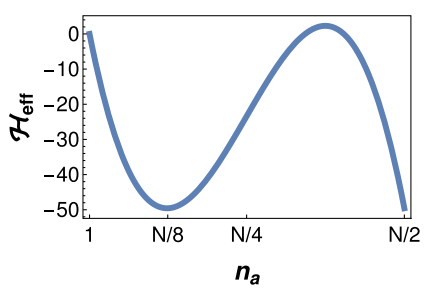

(c) $\kappa=\kappa_{1}$

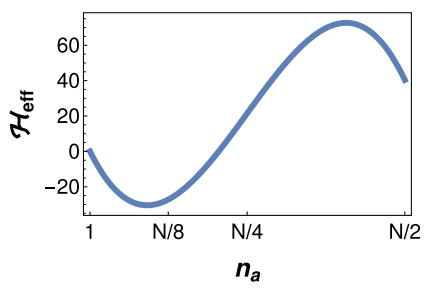

(b) $\kappa=\kappa_{0}+\frac{3}{5}\left(\kappa_{2}-\kappa_{0}\right)$

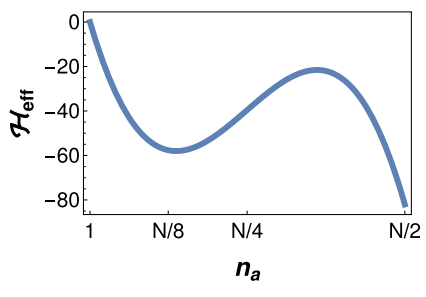

(d) $\kappa=\kappa_{0}+\frac{4}{5}\left(\kappa_{2}-\kappa_{0}\right)$

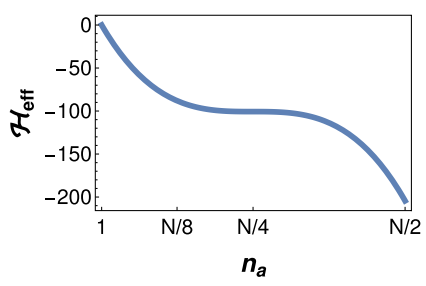

(e) $\kappa=\kappa_{2}$

FIG. 7. $\mathcal{H}_{\text {eff }}$ (in arbitrary units) as a function of the number of antiferro links $n_{a}$, for different values of $\kappa$. In the limit as $\theta \rightarrow 0^{+}$, the probability concentrates in the absolute minimum of $H_{\text {eff. }}$ (a) $\kappa=\kappa_{0}$, the absolute minimum occurs at $n_{a}=1$. (b) $\kappa_{0}<\kappa<\kappa_{1}$ : the absolute minimum shifts to greater values of $n_{a}$, and a new minimum appears at the completely antiferromagnetic configuration $n_{a}=N / 2$. The minima correspond to phases $B+$ and $L$ and the maximum between them to phase $B-$. (c) $\kappa=\kappa_{1}, \mathcal{H}_{\text {eff }}$ is the same at the relative minimum and at the completely antiferromagnetic state with $n_{a}=N / 2$. (d) $\kappa_{1}<\kappa<\kappa_{2}, H_{\text {eff }}$ is lowest for the completely antiferromagnetic configuration. (e) $\kappa=\kappa_{2}$, the relative minimum disappears and the only equilibrium state is the completely antiferromagnetic one.

same value as the completely antiferromagnetic configuration. Finally, at $\kappa_{2}$, this relative minimum disappears and the only stable configuration is that of the absolute minimum for $n_{a}=N / 2$, that is, the completely antiferromagnetic configuration.

The situation described above is illustrated in Fig. 7, in which we plot $\mathcal{H}_{\text {eff }}$ as a function of $n_{a}$, for different values of $\kappa$. Of course, in the large $N$ limit, the values of $\kappa$ at which there are changes in the stability of the solution are in perfect agreement with those obtained from the analysis of the solution of the Euler-Lagrange Eq. (4) for the string profile: $\kappa_{1}$ and $\kappa_{2}$ tend to $\kappa_{t}^{(0)}$ and $\kappa_{M}^{(0)}$, respectively. The completely antiferromagnetic configuration leads to an almost flat, wrinkled, string whereas the completely ferromagnetic distribution corresponds to a buckled configuration, with a definite sign of the curvature. Accordingly, the low temperature phase, comprising antiferromagnetic boundaries and a ferromagnetic bulk yields a buckled string with linear $\left(u^{\prime \prime}=0\right)$ boundaries, as depicted in Figs. 5 and 6. 


\section{CONCLUSIONS}

Despite its simplicity, the 1D spin-string model contains the key ingredients that lead to the emergence of wrinkled and buckled phases in graphene. To keep the model as simplest as possible, the internal degrees of freedom are represented by Ising pseudospins, which stand for the effect of electronic orbital hybridizations and/or charge density fluctuations in graphene. To produce the complex behavior observed in experiments, the couplings between membrane deformations and internal degrees of freedom and the antiferromagnetic interaction between nearest neighbor internal degrees of freedom are essential. Note that the phase diagram of a $2 \mathrm{D}$ version of this model on a hexagonal lattice (numerically obtained in Refs. [10,12]) is completely similar to the 1D case. The main advantage of the $1 \mathrm{D}$ version is its making it possible to derive analytical results, which shed light on the physical reasons underlying the observed behavior.

In our approach, internal degrees of freedom are integrated out to give rise to an effective free energy for the string deformation. This procedure is completely analogous to the one carried out in first-principle models $[8,13]$. Our theory entails that the string curvature is controlled by the local magnetization and the flat string phase $L$ becomes unstable inside a bifurcation line $\kappa_{b}(\theta)$ whose inverse function is two-valued. For a given $\kappa$, lowering the temperature $\theta$ produces buckled string profiles with nonzero global magnetization. For low enough temperatures, the short-ranged antiferromagnetic interaction: (i) modifies the buckled profiles, introducing an antiferromagnetic region close to the boundaries, and (ii) makes the flat string metastable.

The phase diagram of the spin-string system is summarized in Fig. 1 and Table I. In region I, the antiferromagnetic interaction prevails and only the flat phase $L$ exists. In region II, the long-range ferromagnetic interaction dominates and there appears a stable buckled phase $B+$. For each $\theta$ in region III, there is a competition between the ferromagnetic interaction that induces global buckling and the antiferromagnetic interaction that favors the flat phase. Therein, both the flat phase $L$ and the buckled phase $B+$ are locally stable minima of the free energy. In addition, there appears an unstable buckled phase $B-$ that separates these minima.

The above phase diagram is consistent with the numerical findings in a $2 \mathrm{D}$ version of the model, built on a hexagonal lattice to model buckling and rippling in graphene [10]. Key elements in the observed behavior are the existence of a tricritical point $K$, at which all phases coalesce, and a turning point (nose) $N$ in the bifurcation line that splits it into two branches corresponding to high and low temperatures. The existence of these two elements was not investigated in the numerical analysis of the 2D model [10]. However, they may be there because the qualitative shape of this phase diagram is the feature that explains the emergence of the rippled to buckled transition when the system is heated, as recently observed in STM experiments [9]. For small enough antiferromagnetic coupling, $\kappa<\kappa_{\mathrm{n}}$, we may prepare initially the string in the metastable rippled flat profile for low temperatures. As the temperature is increased, the system crosses the lower part of the bifurcation line and suddenly jumps to a buckled state. In light of the above discussion, it is tempting to conjecture that the actual phase diagram of graphene is similar to the one found here.

We expect that our results motivate new investigations of the nontrivial equilibrium states stemming from the nonlinear terms that are present in quantum field models of graphene $[3,4,7,13]$. Both approaches are similar and involve integration of some internal degrees of freedom. Very recently, buckling instabilities in suspended graphene sheets have been attributed to residual stresses produced by the electron-phonon interaction [8]. This conclusion is based on a linear stability analysis of the flat configuration solution of saddle-point equations for phases in thermal equilibrium that were first deduced in Ref. [13]. Whether buckling states bifurcate sub or supercritically from the flat membrane requires a study of small amplitude equations. This problem has not yet been solved but it can be addressed with bifurcation techniques similar to the ones employed in this work.

Our work thus opens new possibilities for further research. It is not yet possible to calibrate parameters of the model to make quantitative comparisons with real experiments in graphene and we have had to settle for qualitative agreement. However, the model simplicity should make it possible to approximately solve its nonequilibrium dynamics, which would allow us to check these new dynamical predictions against recent experiments that probe anomalous dynamical behavior of freestanding graphene [37]. Were this comparison successful, we could be able to calibrate the model parameters so as to make semiquantitative comparisons with experiments.

Summarizing, we expect the mathematical framework described in this paper to motivate and inspire new approaches to the phase diagram of more complex and realistic models. Furthermore, the obtained phase diagram may trigger new experiments. For example, the transition from a rippled to a buckled state would also be set off by decreasing the strength of the short-ranged interaction between the internal degrees of freedom provided its intensity could be controlled. Last, this model could also stimulate both experimental and theoretical research in other physical systems having elastic variables coupled to internal degrees of freedom, such as those considered in Refs. [18-27].

\section{ACKNOWLEDGMENTS}

This work has been supported by the Spanish Ministerio de Economía y Competitividad Grants No. MTM2014-56948C2-2-P (M.R.G. and L.L.B.) and No. FIS2014-53808-P (A.P.). M.R.G. also acknowledges support from Ministerio de Educación, Cultura y Deporte (Spain) through the Formación de Profesorado Universitario program.

\section{APPENDIX A: STABILITY OF THE LOW-TEMPERATURE STRING PROFILES}

At low temperatures $\theta \rightarrow 0^{+}$, the Euler-Lagrange equation becomes equivalent to

$$
\frac{1}{\pi^{2}} u^{\prime \prime}+\operatorname{sgn}(u) \eta(|u|-2 \kappa)=0, u(0)=u(1)=0 .
$$

Both $u$ and $u^{\prime}$ are continuous at the points at which $u=2 \kappa$. When no internal nodes are present, the clamped boundary 
conditions and symmetry considerations imply that there must be two such points $x_{0}$ and $1-x_{0}$. The result is [12]

$$
u^{(0)}(x)= \pm \begin{cases}\frac{2 \kappa x}{x_{0}}, & x<x_{0}, \\ 2 \kappa+\frac{\pi^{2}\left(x-x_{0}\right)\left(1-x_{0}-x\right)}{2}, & x_{0}<x<1-x_{0}, \\ \frac{2 \kappa(1-x)}{x_{0}}, & x>1-x_{0} .\end{cases}
$$

The continuity of the derivative at either $x_{0}$ or $1-x_{0}$ provides the relation between $\kappa$ and $x_{0}$,

$$
\frac{\pi^{2}}{2} x_{0}\left(1-2 x_{0}\right)=2 \kappa \text {. }
$$

Provided $\kappa<\kappa_{M}^{(0)}=\pi^{2} / 32$, there are two solutions $x_{0, j}, j=$ 1,2 , given by Eq. (42), which are symmetrical with respect to $1 / 4, x_{0,1}<1 / 4<x_{0,2}$. For $\kappa>\kappa_{M}^{(0)}$, there are no buckled solutions and $x_{0,1}=x_{0,2}=1 / 4$ if $\kappa=\kappa_{M}^{(0)}$.

Let $u^{(0)}(x)$ be one of these buckled stationary profiles characterized by the sign in Eq. (A2) and the value of $x_{0}$. To study its linear stability, we consider a small disturbance from it, $u(x)=u^{(0)}(x)+\Delta u(x)$. According to the stability conditions described in Sec. II A, we have to solve the linear boundary value problem (BVP):

$$
\begin{array}{r}
\frac{1}{\pi^{2}} \Delta u^{\prime \prime}+\delta\left(u^{(0)}(x)-2 \kappa\right) \Delta u=0 . \\
\Delta u(0)=\Delta u(a)=0, \quad a \leqslant 1 .
\end{array}
$$

Equation (A4a) is the linearization of Eq. (A1) around Eq. (A2) (with positive sign). The profile $u^{(0)}(x)$ is stable if, for any $a \leqslant 1, \Delta u(x) \equiv 0$ is the unique solution of this BVP. On the contrary, if the BVP has a nontrivial solution for some $a<1$, then $u^{(0)}(x)$ is unstable.

Integrating Eq. (A4a) from $x_{J}-$ to $x_{J}+\left(x_{J}\right.$ is either $x_{0}$ or $\left.1-x_{0}\right)$, we find the jump conditions:

$$
\Delta u^{\prime}\left(x_{J}+\right)-\Delta u^{\prime}\left(x_{J}-\right)=-\frac{2}{1-2 x_{0}} \Delta u\left(x_{J}\right) .
$$

As the solution of Eqs. (A4a)-(A4b) is unique up to a multiplicative constant factor, we can fix the slope at $x=0$ to be $\Delta u^{\prime}(0)=1$ [32]. Then $\Delta u\left(0^{+}\right)>0$. If we find $\Delta u(1)<0$, then $\Delta u(a)=0$ at some intermediate point $a \leqslant 1$ and the profile $u^{(0)}(x)$ is unstable.

Equation (A4a) tells us that $\Delta u(x)$ is composed of straight lines, with slope jumps at the points $x_{0}$ and $1-x_{0}$ determined by Eq. (A5). Therefore,

$$
\Delta u= \begin{cases}x, & 0<x<x_{0}, \\ x_{0}+c_{1}\left(x-x_{0}\right), & x_{0}<x<1-x_{0}, \\ x_{0}+c_{1}\left(1-2 x_{0}\right) & \\ \quad+c_{2}\left(x-1+x_{0}\right), & 1-x_{0}<x<1 .\end{cases}
$$

The jump conditions Eq. (A5) readily yield

$$
c_{1}=\frac{1-4 x_{0}}{1-2 x_{0}}, \quad c_{2}=-1 .
$$

Then,

$$
\Delta u(1)=1-4 x_{0} .
$$

Thus, the stationary profile having $x_{0}>1 / 4$, corresponding to $x_{0,2}$ in Eq. (42), produces $\Delta u(1)<0$ and it is unstable as explained above. For the other stationary profile, corresponding to $x_{0,1}<1 / 4, \Delta u(x)$ is positive for $0<x<1$ and the only solution of the BVP Eq. (A4a) is $\Delta u=0$. Therefore, this stationary profile is linearly stable.

\section{APPENDIX B: EFFECTIVE HAMILTONIAN FOR THE PSEUDOSPINS}

We start by deriving the pseudospins' marginal probability $\mathcal{P}(\boldsymbol{\sigma})$ by integrating the canonical distribution $\mathcal{P}(\boldsymbol{u}, \boldsymbol{p}, \boldsymbol{\sigma})$ over the string degrees of freedom. To do so, we rewrite Eq. (1) in matrix form,

$$
\mathcal{H}=\frac{1}{2 m} \boldsymbol{p}^{\boldsymbol{T}} \boldsymbol{p}+\frac{k}{2} \boldsymbol{u}^{\boldsymbol{T}} \boldsymbol{K} \boldsymbol{u}-f \boldsymbol{u}^{\boldsymbol{T}} \boldsymbol{\sigma}+J \boldsymbol{\sigma}^{\boldsymbol{T}} \boldsymbol{J} \boldsymbol{\sigma},
$$

in which $(\boldsymbol{u}, \boldsymbol{p}, \boldsymbol{\sigma})$ are now column matrices of dimension $N,\left(\boldsymbol{u}^{\boldsymbol{T}}, \boldsymbol{p}^{\boldsymbol{T}}, \boldsymbol{\sigma}^{\boldsymbol{T}}\right)$ are their respective transpose matrices, and $\boldsymbol{J}$ and $\boldsymbol{K}$ are symmetric matrices of dimension $N$, namely

$\boldsymbol{J}=\left[\begin{array}{ccccc}0 & \frac{1}{2} & & & \\ \frac{1}{2} & 0 & \frac{1}{2} & & \\ & \frac{1}{2} & 0 & \frac{1}{2} & \\ & & & \ddots & \\ & & & \frac{1}{2} & 0\end{array}\right], \boldsymbol{K}=\left[\begin{array}{ccccc}2 & -1 & & & \\ -1 & 2 & -1 & & \\ & -1 & 2 & -1 & \\ & & & \ddots & \\ & & & -1 & 2\end{array}\right]$.

Also, we make the following change of variables, $\boldsymbol{u}=\boldsymbol{v}+$ $f k^{-1} \boldsymbol{\Lambda} \boldsymbol{\sigma}$, where $\boldsymbol{\Lambda}$ is the inverse of the matrix $\boldsymbol{K}$,

$$
\boldsymbol{\Lambda}_{i j}=\frac{1}{N+1} j(N-i+1)>0, \forall i \geqslant j, \boldsymbol{\Lambda}_{i j}=\boldsymbol{\Lambda}_{j i},
$$

see below for details on the derivation of the elements of $\boldsymbol{\Lambda}$.

Interestingly, the variables $(\boldsymbol{v}, \boldsymbol{p})$ and $\boldsymbol{\sigma}$ become decoupled in the Hamiltonian, making it easy to integrate the canonical distribution over $(\boldsymbol{v}, \boldsymbol{p})$. The result is

$$
\mathcal{P}(\boldsymbol{\sigma}) \propto e^{-\mathcal{H}_{\mathrm{eff}}(\boldsymbol{\sigma}) / \theta}, \mathcal{H}_{\mathrm{eff}}(\boldsymbol{\sigma})=\kappa \boldsymbol{\sigma}^{\boldsymbol{T}} \boldsymbol{J} \boldsymbol{\sigma}-\frac{\pi^{2}}{2 N^{2}} \boldsymbol{\sigma}^{\boldsymbol{T}} \boldsymbol{\Lambda} \boldsymbol{\sigma},
$$

which is Eq. (43) of the main text.

Now, we derive the explicit expression of the elements of the matrix $\boldsymbol{\Lambda}=\boldsymbol{K}^{-1}$. From equation Eq. (B2), we can directly calculate the determinant of the matrix $\boldsymbol{K}_{n}(\boldsymbol{K}$-matrix with dimension $n$ ) as

$$
\begin{aligned}
& \operatorname{det}\left(\boldsymbol{K}_{1}\right)=2, \quad \operatorname{det}\left(\boldsymbol{K}_{2}\right)=3, \\
& \operatorname{det}\left(\boldsymbol{K}_{n}\right)=2 \operatorname{det}\left(\boldsymbol{K}_{n-1}\right)-\operatorname{det}\left(\boldsymbol{K}_{n-2}\right) .
\end{aligned}
$$

Hence,

$$
\operatorname{det}\left(\boldsymbol{K}_{n}\right)=n+1 \text {. }
$$

We take advantage of $\boldsymbol{K}$ being a symmetric matrix $\boldsymbol{K}=$ $\boldsymbol{K}^{\boldsymbol{T}}$, and impose $i \geqslant j$ when calculating $\boldsymbol{\Lambda}_{i j}$, which is also symmetric. Then, for dimension $N$

$$
\begin{aligned}
\boldsymbol{\Lambda}_{i j} & =\frac{1}{N+1}(-1)^{i+j} \operatorname{det}\left(\boldsymbol{K}_{j-1}\right)(-1)^{i-j} \operatorname{det}\left(\boldsymbol{K}_{N-i}\right) \\
& =\frac{1}{N+1} j(N-i+1),
\end{aligned}
$$


where we have made use of

$$
\operatorname{det}\left(\begin{array}{ll}
\boldsymbol{A} & \mathbf{0} \\
\boldsymbol{B} & \boldsymbol{C}
\end{array}\right)=\operatorname{det}(\boldsymbol{A}) \operatorname{det}(\boldsymbol{C}),
$$

in which $\boldsymbol{A}, \boldsymbol{B}$, and $\boldsymbol{C}$ are nonzero matrices and $\mathbf{0}$ the zero matrix.

\section{APPENDIX C: EFFECTIVE HAMILTONIAN LANDSCAPE}

We want to characterize the $\mathcal{H}_{\text {eff }}$ landscape as $\kappa$ is modified, where the phase space is formed by all possible configurations of $\sigma$. For small enough $\kappa$, the completely ferromagnetic configuration with all the pseudospins pointing up (or down) minimizes Eq. (B4). On the other hand, as $\kappa$ increases the configuration minimizing Eq. (B4) changes. Let us start from a completely ordered ferromagnetic configuration $\sigma_{\text {ferro }}$, in which $\sigma_{i}=+1, \forall i$, and change the sign of $\sigma_{l}$, thereby obtaining the configuration $R_{l} \sigma_{\text {ferro }}$. The additional contribution to the free energy is

$$
\begin{aligned}
\Delta \mathcal{H}_{\text {eff }} & \equiv \mathcal{H}_{\text {eff }}\left(R_{l} \sigma_{\text {ferro }}\right)-\mathcal{H}_{\text {eff }}\left(\sigma_{\text {ferro }}\right) \\
& =\frac{\pi^{2}}{2 N^{2}} \sum_{i \neq l}^{N} \Lambda_{l, i}-\kappa
\end{aligned}
$$

where

$$
\sum_{i \neq l}^{N} \boldsymbol{\Lambda}_{l, i}=\frac{(N-1)(N+1-l) l}{2(N+1)} .
$$

This positive expression has a maximum at the center, $l=$ $(N+1) / 2$, and therefore $\Delta \mathcal{H}_{\text {eff }}$ is minimum when the flipping pseudospins are those at the borders of the chain. This suggests that, as $\kappa$ increases, the most probable (minimum free-energy) state will become antiferromagnetic at both boundaries while remaining ferromagnetic in the bulk.

Now we can analyze the behavior of this global minimum with increasing $\kappa$. In light of the discussion above, we restrict ourselves to configurations in which $n_{a}$ consecutive antiferromagnetic links have been created at each boundary; see Fig. 6. We denote by $\mathcal{H}_{\text {eff }}\left(n_{a}\right)$ the value of the effective Hamiltonian for such a configuration. Since $n_{a}$ increases by two, we are interested in evaluating $\mathcal{H}_{\text {eff }}\left(n_{a}\right)-\mathcal{H}_{\text {eff }}\left(n_{a}-2\right)$. Using Eq. (B4), taking into account the symmetries of $\boldsymbol{K}$ and $\boldsymbol{J}$, and that we only have to take care of the terms that change their sign from $\mathcal{H}_{\text {eff }}\left(n_{a}\right)$ to $\mathcal{H}_{\text {eff }}\left(n_{a}-2\right)$, we get the expression

$$
\begin{aligned}
\mathcal{H}_{\mathrm{eff}}\left(n_{a}\right)-\mathcal{H}_{\mathrm{eff}}\left(n_{a}-2\right) & \frac{4 \pi^{2}}{N^{2}}\left[\sum_{j=1}^{n_{a}-1}(-1)^{j} \frac{j\left(N-n_{a}+1\right)}{N+1}\right. \\
& +\sum_{i=n_{a}+1}^{N-n_{a}} \frac{n_{a}(N-i+1)}{N+1} \\
& \left.+\sum_{i=N-n_{a}+2}^{N}(-1)^{i+1} \frac{(N-i+1) n_{a}}{N+1}\right]-8 \kappa .
\end{aligned}
$$

After some simplifications,

$$
\begin{aligned}
\mathcal{H}_{\text {eff }}\left(n_{a}\right)-\mathcal{H}_{\text {eff }}\left(n_{a}-2\right) \\
\quad=\frac{2 \pi^{2}}{N^{2}}\left[-1+\left(1+N-2 n_{a}\right) n_{a}\right]-8 \kappa .
\end{aligned}
$$

Iteration of this recurrence relation gives Eq. (46).

\section{APPENDIX D: STABILITY OF THE PHASES}

Here we determine the stability of the different phases whose approximate profiles near bifurcation points,

$$
u_{S}(x ; C)=C \sin (\pi x),
$$

solve the Euler-Lagrange equations for the total free energy (see Sec. IV). Phase L (flat string profile) has $C=0$, whereas $C \neq 0$ for the buckled phases $B_{ \pm}$. We shall calculate the total free energy for $u_{S}$ as a function of $C$ and determine whether it is a relative maximum or a minimum. The obtained stability results are consistent with the principle of exchange of stabilities in bifurcation theory [28].

The difference of free energies between the sinusoidal and the flat profiles is given by

$$
\Delta F(C ; \kappa, \theta) \equiv \int_{0}^{1} d x\left[f\left(u_{S}, u_{S}^{\prime} ; \kappa, \theta\right)-f_{\mathrm{L}}(\kappa, \theta)\right] .
$$

Note that $\Delta F$ is no longer a functional but a function of the (unknown) amplitude $C$. To simplify our notation, we omit the dependence on $(\kappa, \theta)$ hereafter. Within the same level of approximation as we have been working throughout, we have

$$
\begin{aligned}
\Delta F(C) & \sim \int_{0}^{1} d x\left(\frac{1}{2} \delta f_{2, b} u_{S}^{2}+\frac{1}{4 !} f_{4, b} u_{S}^{4}+\frac{1}{6 !} f_{6, b} u_{S}^{6}\right), \\
& =\frac{1}{4} \delta f_{2, b} C^{2}+\frac{1}{64} f_{4, b} C^{4}+\frac{1}{2304} f_{6, b} C^{6},
\end{aligned}
$$

where $\delta f_{2, b}=f_{2}-f_{2, b}, f_{n, b}$ is the value of $f_{n}$ over the bifurcation curve, as introduced in Sec. IV, and we have neglected $O\left(C^{8}\right)$ terms. The equilibrium values of $C$, which we denote by $C_{\text {eq }}$, are found by seeking the extrema of $\Delta F(C)$, see below.

Far from the critical point, consistently with the procedure for solving perturbatively the Euler-Lagrange equation in Sec. IV, the term proportional to $C^{6}$ in Eq. (D3) can be neglected. Then, the nonvanishing values of $C_{\text {eq }}$ obey

$$
C_{\mathrm{eq}}^{2} \sim-\frac{8 \delta f_{2, b}}{f_{4 b}} .
$$

Note that $\delta f_{2, b}$ is of the order of $\epsilon^{2}$, cf. Eqs. (22) and (21). Thus, $C_{\text {eq }}$ is $O(\epsilon)$ and with the substitution $C_{\text {eq }}=\epsilon A$, the above equation is completely equivalent to Eq. (29). Insertion of $C_{\text {eq }}$ into Eq. (D3) gives the free energy difference between the buckled and the flat phase,

$$
\Delta F_{\mathrm{eq}} \sim-\frac{\delta f_{2, b}^{2}}{f_{4, b}} .
$$

which shows that the sign of $\Delta F_{\text {eq }}$ is controlled by the sign of $f_{4, b}$.

The stability of the phases can be further elucidated by looking at the sign of the second derivative of $\Delta F$ with respect to $C$, which is given by

$$
\frac{\partial^{2} \Delta F}{\partial C^{2}} \sim \frac{\delta f_{2, b}}{2}+\frac{3}{16} f_{4 b} C^{2}
$$


Therefore,

$$
\left.\frac{\partial^{2} \Delta F}{\partial C^{2}}\right|_{\mathrm{eq}}=-\delta f_{2, b},
$$

and the stability is controlled by the sign of $\delta f_{2, b}$. We recall that $f_{4, b}$ vanishes at the critical point $\kappa$, and that $f_{4, b}>0\left(f_{4, b}<0\right)$ above (below) it. Then, above the critical point, the phase $B+$ bifurcates inside the bifurcation line $\left(\delta f_{2, b}<0\right)$ where the flat phase L becomes unstable, and is thus stable: $\left.\partial_{C}^{2} F(C)\right|_{B+}>0$ and, consistently, $\left.\Delta F_{\text {eq }}\right|_{B+}<0$. To the right of the critical point, the phase $B$ - emerges outside the bifurcation line $\left(\delta f_{2, b}>0\right)$, where the flat phase is stable, and is unstable: $\left.\partial_{C}^{2} F(C)\right|_{B-}<0$ and $\left.\Delta F_{\text {eq }}\right|_{B-}>0$. The phase $B-$ is indeed unstable but it does not correspond to a (local) maximum of the free-energy functional, but to some kind of "saddle point" extremum that is neither a minimum nor a maximum [38].

In the vicinity of the tricritical point $K$, we have to keep the $C^{6}$ terms, and substitute the coefficients of $\Delta F(C)$ with their leading behaviors. With the same notation as before,

$$
\Delta F(C) \sim \frac{1}{4} \delta f_{2, c} C^{2}+\frac{1}{64} f_{4, c}^{(1)} C^{4}+\frac{1}{2304} f_{6, c} C^{6},
$$

where

$$
\delta f_{2, c}=\epsilon^{4} \varphi_{4, c}, \quad f_{4, c}^{(1)}=\epsilon^{2} 6 \sqrt{3} \chi, \quad f_{6, c}=36,
$$

and we have used Eqs. (21) (with $\kappa_{2}=\theta_{2}=0$ ), (30), and (32). Again, $C_{\mathrm{eq}}$ is found by looking for the extrema of $\Delta F$, and $C_{\text {eq }}=O(\epsilon)$. By introducing $C_{\text {eq }}=\epsilon A$, we have that $A$ is the solution of the biquadratic Eq. (35). Let us denote by $A_{ \pm}^{2}$ the two solutions of Eq. (35), with $A_{+}^{2}>A_{-}^{2}$. As discussed in Sec. IV, (i) above the critical point, $\chi>0$, it is only $A_{+}^{2}$ that makes sense $\left(A_{-}^{2}<0\right)$ and (ii) below the critical point, $\chi<0$, both $A_{+}^{2}$ and $A_{-}^{2}$ are positive in a certain domain.

Again, the local stability of the phases is given by the second derivative of $\Delta F$ at equilibrium. After a little algebra, one gets the result

$$
\left.\frac{\partial^{2} \Delta F}{\partial C^{2}}\right|_{B \pm}= \pm \frac{\sqrt{3}}{4} \epsilon^{6} \tilde{A}_{ \pm}^{2} \sqrt{9 \chi^{2}-4 \varphi_{4, c}} .
$$

Then the phase $\mathrm{B}+$ is locally stable and the phase $\mathrm{B}-$ is unstable within their respective domains of existence. Below the critical point, we recall that the phase $\mathrm{B}+$ exists for $\kappa<$ $\kappa_{M}(\theta)$, where $\kappa_{M}(\theta)$ is the first-order line given by Eq. (36), whereas the phase $\mathrm{B}-$ only exists between the bifurcation line and the first-order line, $\kappa_{b}(\theta)<\kappa<\kappa_{M}(\theta)$. Over $\kappa_{M}(\theta)$, both phases $B \pm$ merge, disappear and $\partial^{2} \Delta F /\left.\partial C^{2}\right|_{B \pm}=0$, because the argument of the square root becomes equal to zero. Above the critical point, only the plus sign is possible and Eq. (D10) smoothly matches with Eq. (D7).

Let us focus on region III of the phase diagram in Fig. 1, that is, between the bifurcation and the first-order line, $\kappa_{b}(\theta)<$ $\kappa<\kappa_{M}(\theta)$. Further analysis is necessary to find out which of the two locally stable phases, the flat $L$ phase and the buckled $B+$ phase, gives the absolute minimum of the free energy. The free-energy difference $\Delta F$ is obtained by inserting $C_{\mathrm{eq}}=\epsilon A$ in Eq. (D8), which yields

$$
\Delta F_{B \pm}=\frac{\epsilon^{6}}{48} \tilde{A}_{ \pm}^{2}\left[8 \varphi_{4, c} \pm 3 \chi \sqrt{9 \chi^{2}-4 \varphi_{4, c}}-9 \chi^{2}\right] .
$$

Recall that $\chi<0$ below the critical point, and thus $\sqrt{9 \chi^{2}}=$ $-3 \chi$. Consistently with its unstable character, $\Delta F_{B-} \geqslant 0$, it varies from $\Delta F_{B-}=0$ over the bifurcation line $\kappa_{b}(\theta)$, at which $A_{-}$vanishes, to the positive value $\Delta F_{B}^{\max }=9 \epsilon^{6} \tilde{A}^{2} \chi^{2} / 48>0$ at the first-order line $\kappa_{M}(\theta)$. On the other hand, $\Delta F_{B+}<0$ at the bifurcation line, whereas $\Delta F_{B+}=\Delta F_{B}^{\max }>0$ at the first-order line because the phases $B \pm$ merge. Thus, there must be a coexistence line at which $\Delta F_{B+}$ vanishes and phases $B+$ and $L$ are equally probable. Equation (D11) determines the condition $\varphi_{2, c}=27 \chi^{2} / 16$ or

$$
\kappa_{t}(\theta)=\kappa_{b}(\theta)+\frac{27 \sqrt{3}}{96}\left(\theta-\theta_{c}\right)^{2}, \theta<\theta_{c},\left|\theta-\theta_{c}\right| \ll 1 .
$$

For $\kappa_{b}(\theta)<\kappa<\kappa_{t}(\theta)$, the most stable phase is $\mathrm{B}+$, whereas the flat phase $\mathrm{L}$ is metastable; the situation is just reversed in the region $\kappa_{t}(\theta)<\kappa<\kappa_{M}(\theta)$.
[1] J. C. Meyer, A. K. Geim, M. I. Katsnelson, K. S. Novoselov, T. J. Booth, and S. Roth, Nature 446, 60 (2007).

[2] A. Fasolino, J. H. Los, and M. I. Katsnelson, Nat. Mater. 6, 858 (2007).

[3] D. Gazit, Phys. Rev. B 79, 113411 (2009).

[4] P. San-Jose, J. Gonzalez, and F. Guinea, Phys. Rev. Lett. 106, 045502 (2011).

[5] L. L. Bonilla and A. Carpio, Phys. Rev. B 86, 195402 (2012).

[6] L. L. Bonilla and A. Carpio, J. Stat. Mech.: Theor. Exp. (2012) P09015.

[7] J. González, Phys. Rev. B 90, 165402 (2014).

[8] L. L. Bonilla and M. Ruiz-Garcia, Phys. Rev. B 93, 115407 (2016).

[9] J. K. Schoelz, P. Xu, V. Meunier, P. Kumar, M. Neek-Amal, P. M. Thibado, and F. M. Peeters, Phys. Rev. B 91, 045413 (2015).

[10] M. Ruiz-Garcia, L. L. Bonilla, and A. Prados, Phys. Rev. B 94, 205404 (2016).
[11] L. L. Bonilla, A. Carpio, A. Prados, and R. R. Rosales, Phys. Rev. E 85, 031125 (2012).

[12] M. Ruiz-García, L. L. Bonilla, and A. Prados, J. Stat. Mech.: Theor. Exp. (2015) P05015.

[13] F. Guinea, P. Le Doussal, and K. J. Wiese, Phys. Rev. B 89, 125428 (2014).

[14] P. O. Lehtinen, A. S. Foster, A. Ayuela, A. Krasheninnikov, K. Nordlund, and R. M. Nieminen, Phys. Rev. Lett. 91, 017202 (2003).

[15] A. V. Krasheninnikov, K. Nordlund, P. O. Lehtinen, A. S. Foster, A. Ayuela, and R. M. Nieminen, Carbon 42, 1021 (2004).

[16] A. Prados, L. L. Bonilla, and A. Carpio, J. Stat. Mech.: Theor. Exp. (2010) P06016.

[17] L. L. Bonilla, A. Prados, and A. Carpio, J. Stat. Mech.: Theor. Exp. (2010) P09019.

[18] A. Prados, A. Carpio, and L. L. Bonilla, Phys. Rev. E 86, 021919 (2012). 
[19] J. Kanamori, J. Appl. Phys. 31, S14 (1960).

[20] E. Pytte, Phys. Rev. B 8, 3954 (1973).

[21] J. Feder and E. Pytte, Phys. Rev. B 8, 3978 (1973).

[22] P. A. Rikvold, Z. Phys. B 26, 195 (1977).

[23] P. A. Rikvold, Bussei Kenkyu 33, E43 (1980).

[24] T. Schneider and E. P. Stoll, Phys. Rev. Lett. 31, 1254 (1973).

[25] T. Schneider and E. Stoll, Phys. Rev. B 17, 1302 (1978).

[26] F. J. Pérez-Reche, L. Truskinovsky, and G. Zanzotto, Phys. Rev. Lett. 99, 075501 (2007).

[27] F. J. Pérez-Reche, L. Truskinovsky, and G. Zanzotto, Phys. Rev. Lett. 101, 230601 (2008).

[28] G. Iooss and D. D. Joseph, Elementary Stability and Bifurcation Theory, 2nd ed. (Springer, Berlin, 1990).

[29] L. D. Landau and E. M. Lifshitz, Statistical Physics, Part 1. Course of Theoretical Physics, 3rd ed. (Elsevier ButterworthHeinemann, Oxford, 1980), Vol. 5.

[30] J. Yeomans, Statistical Mechanics of Phase Transitions (Oxford Science Publications, Oxford, 1993).

[31] R. J. Glauber, J. Math. Phys. 4, 294 (1963).
[32] I. M. Gelfand and S. V. Fomin, Calculus of Variations (Dover, New York, 2000).

[33] We use L (from level) instead of $F$, to avoid confusion in sentences such as the free energy $F$ of the phase $F$.

[34] R. B. Griffiths, J. Chem. Phys. 60, 195 (1974).

[35] S. Sarbach and M. E. Fisher, Phys. Rev. B 20, 2797 (1979).

[36] In Ref. [12], it was incorrectly stated that this configuration was metastable, but this does not affect Ref. [12] results and conclusions. The instability of the configuration for $x_{0,2}$ can also be shown from the pseudospins point of view; see discussion below.

[37] M. L. Ackerman, P. Kumar, M. Neek-Amal, P. M. Thibado, F. M. Peeters, and S. Singh, Phys. Rev. Lett. 117, 126801 (2016).

[38] Note that the phase B + cannot be produced by an expansion around the flat solution below the critical point, because its amplitude does not vanish at the bifurcation line. This is the reason why the solution obtained to the right of the critical point corresponds to the phase $\mathrm{B}-$. 